\title{
Recent advances in extraction and analysis procedures of natural chlorogenic acids
}

\author{
Dorota Wianowska • Marta Gil
}

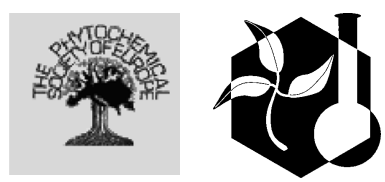

Received: 26 February 2018/ Accepted: 4 October 2018/Published online: 10 October 2018

(C) The Author(s) 2018

\begin{abstract}
Chlorogenic acids (CQAs), the esters of caffeic and quinic acid, are biologically important phenolic compounds present in many plant species. Nowadays the knowledge about their pro-health properties including anti-cancer activity is great. Yet the supposition that they may be helpful in fighting obesity and modify glucose-6-phosphatase involved in glucose metabolism has led to some revival of research on CQAs properties and their natural occurrence. Considering the growing interest in natural products rich in CQAs, it is not surprising that more efficient extraction methods, leading to obtained extracts with increased CQAs content, are currently being sought. It is worth mentioning here that this task is not very easy since these compounds readily degrade/transform to others. Much attention is also paid to the proper analysis of CQAs content in plants and plant products due to the fact that the main CQAs representative in nature i.e. 5-O-caffeoylquinic acid (5-CQA) is commonly employed as a marker in the quality control of various natural products. Yet due to their structural similarity and instability, and the fact that the number of commercially available standards of CQAs is limited to just a few, the correct analysis of these compounds in plants is another challenge that
\end{abstract}

D. Wianowska $(\bowtie) \cdot$ M. Gil

Department of Chromatographic Methods, Faculty of

Chemistry, Maria Curie-Skłodowska University, Pl. Maria

Curie-Skłodowska 3, 20-031 Lublin, Poland

e-mail: dorota.wianowska@poczta.umcs.lublin.pl should be faced. The aim of this review is to summarize the recent advances in the chemical analysis of chlorogenic acids, including their natural sources, properties, and methods that have been developed to improve CQAs extraction and analysis, with special attention paid to 5-CQA as the compound of the CQAs family with many beneficial effects on human health.

Keywords 5-CQA · Isolation · LC-MS/MS · Identification $\cdot$ Fragmentation patterns $\cdot$ Plant analysis

$\begin{array}{ll}\text { Abbreviations } & \\ \text { 1,3-diCQA } & \text { 1,3-Di- } O \text {-caffeoylquinic acid } \\ \text { 1-CQA } & \text { 1- } O \text {-Caffeoylquinic acid } \\ \text { 3,4-diCQA } & \text { 3,4-di- } O \text {-Caffeoylquinic acid } \\ \text { 3,5-diCQA } & \text { 3,5-di- } O \text {-Caffeoylquinic acid } \\ \text { 3-CQA } & \text { 3- } O \text {-Caffeoylquinic acid } \\ \text { 4,5-diCQA } & \text { 4,5-di- } O \text {-Caffeoylquinic acid } \\ \text { 4-CQA } & \text { 4- } O \text {-Caffeoylquinic acid } \\ \text { 5-CQA } & \text { 5-O-Caffeoylquinic acid } \\ \text { ASE } & \text { Accelerated solvent extraction } \\ \text { CA } & \text { Caffeic acid } \\ \text { CE } & \text { Capillary electrophoresis } \\ \text { CE-ED } & \text { Capillary electrophoresis with } \\ \text { CFQAs } & \text { electrochemical detection } \\ \text { CQAs } & \text { Caffeoylferuloylquinic acids } \\ \text { CSiQAs } & \text { Chlorogenic acids } \\ \text { DAD } & \text { Diode array detection }\end{array}$




\begin{tabular}{|c|c|}
\hline diCQAs & di-Esters of caffeic acid \\
\hline ESE & Enhanced solvent extraction \\
\hline ESI & Electrospray ionization \\
\hline FL & Fluorescence \\
\hline FQAs & Feruloylquinic acids \\
\hline FUASE & $\begin{array}{l}\text { Focused ultrasound assisted solvent } \\
\text { extraction }\end{array}$ \\
\hline FUSLE & $\begin{array}{l}\text { Focused ultrasound solid-liquid } \\
\text { extraction }\end{array}$ \\
\hline IP & Ion trap \\
\hline LC & Liquid chromatography \\
\hline MASE & $\begin{array}{l}\text { Microwave assisted solvent } \\
\text { extraction }\end{array}$ \\
\hline MEEKC & $\begin{array}{l}\text { Microemulsion electrokinetic } \\
\text { chromatography }\end{array}$ \\
\hline MEKC & $\begin{array}{l}\text { Micellar electrokinetic } \\
\text { chromatography }\end{array}$ \\
\hline MRM & Multiple reaction monitoring \\
\hline MS & Mass spectroscopy \\
\hline MSPD & $\begin{array}{l}\text { Matrix solid-phase dispersion } \\
\text { technique }\end{array}$ \\
\hline NIR & Near-infrared spectroscopy \\
\hline NMR & Nuclear magnetic resonance \\
\hline$p$ CoQAs & $p$-Coumaroylquinic acids \\
\hline PDA & Photodiode array detection \\
\hline PHWE & Pressurized hot water extraction \\
\hline PLE & Pressurized liquid extraction \\
\hline PSE & Pressurized solvent extraction \\
\hline QA & Quinic acid \\
\hline ROS & Reactive oxygen species \\
\hline SCE & Saturated calomel electrode \\
\hline SCF & Supercritical fluid \\
\hline SFE & Supercritical fluid extraction \\
\hline SPE & Solid phase extraction \\
\hline SRM & Selected reaction monitoring \\
\hline SSDM & Sea sand disruption method \\
\hline SWE & $\begin{array}{l}\text { Subcritical water extraction or } \\
\text { superheated water extraction }\end{array}$ \\
\hline tetraCQA & tetra-Ester of caffeic acid \\
\hline TOF & Time of flight \\
\hline TQD & Triple quadrupole \\
\hline triCQAs & tri-Esters of caffeic acid \\
\hline UASE & $\begin{array}{l}\text { Ultrasound-assisted solvent } \\
\text { extraction }\end{array}$ \\
\hline UPLC & $\begin{array}{l}\text { Ultra-performance liquid } \\
\text { chromatography }\end{array}$ \\
\hline UV & Ultraviolet \\
\hline
\end{tabular}

\section{Introduction}

Chlorogenic acids (CQAs) are naturally occurring phenolic compounds found in all higher plants (Clifford 1999, 2000). Due to their various biological properties, such as antispasmodic (Farah et al. 2005), antioxidant (Kweon et al. 2001), inhibition of the HIV1 integrase (Kwon et al. 2000) and mutagenicity of the carcinogenic compounds (Farah et al. 2005), they are very important plant secondary metabolites. In the light of the pharmacological results (Ludwig et al. 2014; Johnston et al. 2003), they are also supposed to be helpful in fighting obesity and modify glucose-6phosphatase involved in glucose metabolism. These assumptions, although unproven, were sufficient for some revival of research on CQAs properties and their natural occurrence, the more so because new applications of CQAs are proposed in pharmaceuticals, foodstuffs, food additives and cosmetics.

From the chemical point of view CQAs constitute a large family of esters formed between quinic acid and one or more trans-hydroxycinnamic acid derivatives, most commonly caffeic, $p$-coumaric, and ferulic ones (Clifford 2000). Taking into account the identity, number and position of the acyl residues in this group of compounds there can be distinguished:

- Mono-esters of caffeic, p-coumaric and ferulic acid: caffeoylquinic acids (CQAs),

- $p$-coumaroylquinic acids (pCoQAs) and feruloylquinic acids (FQAs), respectively.

- Di-esters, tri-esters and the single tetra-ester of caffeic acid: diCQAs, triCQAs and tetraCQA, respectively.

- Mixed di-esters of caffeic and ferulic acid or caffeic and sinapic acid: caffeoylferuloylquinic acids (CFQAs) and caffeoylsinapoylquinic acids (CSiQAs).

- Mixed esters involving various permutations of one or three caffeic acid residues with one or two dibasic aliphatic acid residues e.g. glutaric, oxalic, succinic.

Figure 1 presents the general structures of monoand dicaffeoylquinic, p-coumaroylquinic, and feruloylquinic acids together with the structures of the most characteristic representatives of mono- and dicaffeoylquinic acids for plants i.e. 5-O-caffeoylquinic and 1,3-di- $O$-caffeoylquinic acids. 
Fig. 1 Structures of monoand dicaffeoylquinic, p-coumaroylquinic, and feruloylquinic acids<smiles>[R6]OC1CCC(OCC)(C(=O)O)CC([R6])C1[R6]</smiles>

Quinic acid

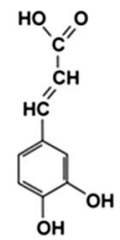

Caffeic acid

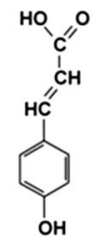

$P$-coumaric acid

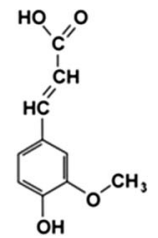

Ferulic acid

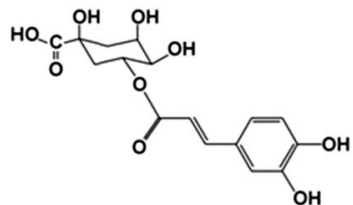

5-O-caffeoylquinic acid

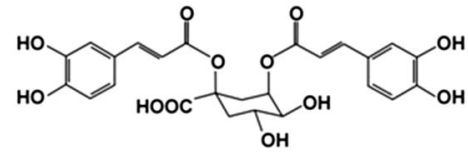

1,3-di-O-caffeoylquinic acid

\begin{tabular}{|c|c|c|c|c|c|c|}
\hline No. & Compound name & Abbreviation & $\mathbf{R}_{1}$ & $\mathbf{R}_{3}$ & $R_{4}$ & $\mathrm{R}_{\mathbf{s}}$ \\
\hline $\mathbf{l}$ & 1-O-caffeoylquinic acid & $1-\mathrm{CQA}$ & $\mathrm{C}$ & $\mathrm{H}$ & $\mathrm{H}$ & $\mathrm{H}$ \\
\hline 2 & 3-O-caffeoylquinic acid (neochlorogenic acid) & 3-CQA & $\mathrm{H}$ & $\mathrm{C}$ & $\mathrm{H}$ & $\mathrm{H}$ \\
\hline 3 & 5-O-caffeoylquinic acid (chlorogenic acid) & 5-CQA & $\mathrm{H}$ & $\mathrm{H}$ & $\mathrm{H}$ & $\mathrm{C}$ \\
\hline 4 & 4-O-caffeoylquinic acid (cryptochlorogenic acid) & 4-CQA & $\mathrm{H}$ & $\mathrm{H}$ & $\mathrm{C}$ & $\mathrm{H}$ \\
\hline 5 & 1,3-di-O-caffeoylquinic acid & 1,3-diCQA & $\mathrm{C}$ & $\mathrm{C}$ & $\mathrm{H}$ & $\mathrm{H}$ \\
\hline 6 & 1,4-di-O-caffeoylquinic acid & 1,4-diCQA & $\mathrm{C}$ & $\mathrm{H}$ & $\mathrm{C}$ & $\mathrm{H}$ \\
\hline 7 & 1,5-di-O-caffeoylquinic acid & 1,5-diCQA & $\mathrm{C}$ & $\mathrm{H}$ & $\mathrm{H}$ & $\mathrm{C}$ \\
\hline 8 & 3,4-di-O-caffeoylquinic acid & 3,4-diCQA & $\mathrm{H}$ & $\mathrm{C}$ & $\mathrm{C}$ & $\mathrm{H}$ \\
\hline 9 & 3,5-di-O-caffeoylquinic acid & 3,5-diCQA & $\mathrm{H}$ & $\mathrm{C}$ & $\mathrm{H}$ & $\mathrm{C}$ \\
\hline 10 & 4,5-di-O-caffeoylquinic acid & 4,5-diCQA & $\mathrm{H}$ & $\mathrm{H}$ & $\mathrm{C}$ & $\mathrm{C}$ \\
\hline 11 & 1-O-p-coumaroylquinic acid & 1-p $\mathrm{CoQA}$ & $p \mathrm{Co}$ & $\mathrm{H}$ & $\mathrm{H}$ & $\mathrm{H}$ \\
\hline 12 & 3-O-p-coumaroylquinic acid & $3-p \mathrm{CoQA}$ & $\mathrm{H}$ & $p \mathrm{Co}$ & $\mathrm{H}$ & $\mathrm{H}$ \\
\hline 13 & 5-O-p-coumaroylquinic acid & 5-p $\mathrm{CoQA}$ & $\mathrm{H}$ & $\mathrm{H}$ & $\mathrm{H}$ & $p \mathrm{Co}$ \\
\hline 14 & 4-O-p-coumaroylquinic acid & 4-p CoQA & $\mathrm{H}$ & $\mathrm{H}$ & $p C o$ & $\mathrm{H}$ \\
\hline 15 & 1,3 -di-O-p-coumaroylquinic acid & 1,3-dip CoQA & $p \mathrm{Co}$ & $p \mathrm{Co}$ & $\mathrm{H}$ & $\mathrm{H}$ \\
\hline 16 & 1,4-di-O-p-coumaroylquinic acid & 1,4-dip $\mathrm{CoQA}$ & $p \mathrm{Co}$ & $\mathrm{H}$ & $p \mathrm{Co}_{0}$ & $\mathrm{H}$ \\
\hline 17 & 1,5 -di- $O$-p-coumaroylquinic acid & 1,5-dipCoQA & $p \mathrm{Co}$ & $\mathrm{H}$ & $\mathrm{H}$ & $p \mathrm{Co}$ \\
\hline 18 & 3,4-di-O-p-coumaroylquinic acid & 3,4-dip $\mathrm{CoQA}$ & $\mathrm{H}$ & $p \mathrm{Co}$ & $p \mathrm{Co}$ & $\mathrm{H}$ \\
\hline 19 & 3,5-di-O-p-coumaroylquinic acid & 3,5-dip $\mathrm{CoQA}_{0}$ & $\mathrm{H}$ & $p \mathrm{Co}$ & $\mathrm{H}$ & $p \mathrm{Co}$ \\
\hline 20 & 4,5-di-O-p-coumaroylquinic acid & 4,5-dip CoQA & $\mathrm{H}$ & $\mathrm{H}$ & $p \mathrm{Co}$ & $p \mathrm{Co}$ \\
\hline 21 & 1-O-feruloylquinic acid & 1-FQA & F & $\mathrm{H}$ & $\mathrm{H}$ & $\mathrm{H}$ \\
\hline 22 & 3-O-fenuloylquinic acid & 3-FQA & $\mathrm{H}$ & $\mathbf{F}$ & $\mathrm{H}$ & $\mathrm{H}$ \\
\hline 23 & 5-O-feruloylquinic acid & 5-FQA & $\mathrm{H}$ & $\mathrm{H}$ & $\mathrm{H}$ & $F$ \\
\hline 24 & 4-O-fenuloylquinic acid & 4-FQA & $\mathrm{H}$ & $\mathrm{H}$ & $\mathrm{F}$ & $\mathrm{H}$ \\
\hline 25 & 1,3-di-O-feruloylquinic acid & 1,3-diFQA & F & F & $\mathrm{H}$ & $\mathrm{H}$ \\
\hline 26 & 1,4-di-O-feruloylquinic acid & 1,4-diFQA & $F$ & $\mathrm{H}$ & $\mathrm{F}$ & $\mathrm{H}$ \\
\hline 27 & 1,5-di-O-feruloylquinic acid & 1,5-diFQA & $\mathrm{F}$ & $\mathrm{H}$ & $\mathrm{H}$ & $F$ \\
\hline 28 & 3,4-di-O-feruloylquinic acid & 3,4-diFQA & $\mathrm{H}$ & F & $\mathrm{F}$ & $\mathrm{H}$ \\
\hline 29 & 3,5-di-O-feruloylquinic acid & 3,5-diFQA & $\mathrm{H}$ & $\mathrm{F}$ & $\mathrm{H}$ & $\mathrm{H}$ \\
\hline 30 & 4,5-di-O-fenuloylquinic acid & 4,5-diFQA & $\mathrm{H}$ & $\mathrm{H}$ & $\mathrm{F}$ & $\mathrm{F}$ \\
\hline
\end{tabular}

Q- quinic acid, C-caffeic acid, $p$ Co- $p$-coumaric acid, F- fenulic acid 
One of the significant features of CQAs is their chemical instability and formation/degradation to other compounds, including chlorogenic acids representatives (Shearer et al. 2003; Jaiswal et al. 2012; Wianowska et al. 2015a, b; Dawidowicz and Typek 2010, 2017). According to Dawidowicz and Typek (2010), the conventional heating of 5-O-caffeoylquinic acid (5-CQA) in the presence of water causes its isomerization and transformation. In Dawidowicz and Typek (2017) it was proved that 5-CQA does not only isomerize to 3-O-caffeoylquinic acid (3CQA) and 4- $O$-caffeoylquinic acid (4-CQA), but also undergoes other transformations such as esterification and reaction with water i.e. hydrolysis and/or addition of water molecule to the double bond. These processes take place not only in the CQAs solutions but also during their isolation from plant materials and they could lead to getting extracts with a lower content of biologically active phenolic compounds.

Recently there has been an increased interest in natural products rich in chlorogenic acids. Consequently, more efficient extraction methods, leading to getting extracts with a greater CQAs content and a reduced content of their degradation products, are being sought. Searching for new and alternative methodologies of CQAs extraction from plants is additionally supported by the fact that some of those compounds can be found in nature at very low concentrations. Thus, to obtain their sufficient amounts very effective isolation methods are needed, the more that their structural diversity and complexity make chemical synthesis rather unprofitable. Unfortunately, the elimination/limitation of CQAs degradation/transformation is not the only problem in the CQAs determination in plants or plant products. The number of compounds needed to be identified during a single analysis is steadily increasing, though the number of commercially available standards is limited to just a few. The large number of CQAs with very similar properties and their tendency for ready formation of transformation/degradation products during their extraction, makes the CQAs analysis in natural samples complicated. This has been accomplished more and more often by means of hyphenated techniques particularly using LC-MS ${ }^{\mathrm{n}}$ (Clifford and Walker 1987; Clifford and Ramirez-Martinez 1990; Clifford et al. 2005, 2010; Jaiswal et al. 2011, 2012; Wianowska et al. 2015a, b; Dawidowicz and Typek 2010, 2017; Deshpande et al. 2014).
The aim of this review is to summarize the recent advances in the chemical analysis of chlorogenic acids, including their natural sources, properties, and methods that have been developed to improve CQAs extraction and analysis, with special attention paid to 5-CQA as the compound of the CQAs family with many beneficial effects on human health.

\section{CQAs occurrence}

CQAs belong to very common plant constituents. They are present, for example, in apples, stone fruits (peaches, nectarines, plums, lychees, mangoes, cherries), berry fruits (blueberries, blackcurrants, blackberries, bilberries), brassica vegetables (kale, cabbage and Brussels sprouts), apiaceae (celery, carrots, caraway and coriander), and other miscellaneous vegetables like corn salad, anise stars and potato. Nevertheless, despite their high prevalence, they are believed to be the most characteristic constituents of plants from the Asteraceae family, such as artichoke (Cynara scolymus L.), black salsify (Scorzonera hispanica L.), purple coneflower (Echinacea purpurea L. Moench), common yarrow (Achillea millefolium L.), milk thistle (Silybum marianum L. Gaertner), coltsfoot (Tussilago farfara L.), tansy (Tanacetum vulgare L.), chamomile (Matricaria chamomilla L.) (Clifford 1999, 2000; Lattanzio et al. 2005, 2009; Upadhyay and Rao 2013; Abu-Reidah et al. 2013; Mazzafera 1997). Yet even if these compounds are the most characteristic of the plant family, the CQAs content is very differentiated. According to Lattanzio et al. $(2005,2009)$, the total content of CQAs in the artichoke ranges from $8 \%$ on dry matter basis in young artichoke tissues to less than $1 \%$ in the senescent ones. Of the total content of the CQAs in this plant, 5-CQA is the most represented single component (39\% of all CQAs in the artichoke), followed by 1,5-diCQA (21\%) and 3,4-diCQA (11\%). The content of 1,3diCQA is $1.5 \%$ of all CQAs. In contrast, black salsify provides $180 \mathrm{mg}$ per $\mathrm{kg}$ of dry matter of 5-CQA with little or none content of the other CQA derivatives (Upadhyay and Rao 2013).

CQAs are present in all parts of plants (seeds, roots, tubers, leaves and flowers) as well as in different products prepared from them, particularly in beverages, such as coffee, green and black tea, yerba mate, juice, infusion and even wine. It should be 
emphasized, however, that the CQAs qualitative and quantitative composition is various and dependent not only on plant parts but also on the plant physiological stage, conditions of storage and plants processing, especially when the processing is accompanied by heat and/or enzymatic treatment. One should also keep in mind the influence of UV radiation. Although trans isomers of chlorogenic acids occur predominantly in plants, cis CQAs isomers are produced by UVirradiation. Clifford observed in (Clifford et al. 2008) that the extract of coffee leaves contains a greater amount of cis-5-O-caffeoylquinic acid compared to the trans isomer than the extract of green coffee beans and concluded that the greater content of cis-5-CQA can be assigned to the more intense UVirradiation to which leaves are naturally exposed.

Whole apples have been reported to contain CQAs in the range 62-358 $\mathrm{mg}$ per kg of fresh matter (Spanos and Wrolstad 1992). They are found at similar concentrations in the apple's isolated flesh and skin (mean values 30-60 mg per $\mathrm{kg}$ ) but are absent in the seeds and pomace (Burda et al. 1998). With the change of apples variety not only the total CQAs content but also the CQAs composition changes. Yet regardless of the apples variety, 5-CQA is the main constituent. Variability of quantitative and qualitative compositions of CQAs is also characteristic of juices and ciders. In commercially available juices, the CQAs content can be from the undetectable level up to 208 $\mathrm{mg}$ per litre of 5-CQA alone, and in ciders in the range of 11-480 mg per litre or even higher when these are produced from the fruit of Sorbus domestica $\mathrm{L}$. known for a high 5-CQA content (1500 mg per $\mathrm{kg}$ ) (Ritter et al. 1995).

The mean CQAs content in stone fruits is in the range $150-600 \mathrm{mg}$ per $\mathrm{kg}$ of fresh matter. Compared with apples, in the fresh stone fruits 3-CQA is more prominent compared to 5-CQA. However, in peach and apricot juice the 5-CQA level is about 3-4 times greater than 3-CQA with the total CQAs content 9 and $4 \mathrm{mg}$ per litre, respectively (Clifford 1999).

One of the richest dietary sources of CQAs are coffee beans. Depending on the species, green coffee beans contain 6-12\% CQAs for the dry weight of the coffee beans. During roasting there is progressive degradation and transformation of CQAs. It is estimated that $8-10 \%$ CQAs are lost for every $1 \%$ of the dry weight of the total CQAs amount in the coffee beans. Clifford and Walker (1987) evaluated that a
$200 \mathrm{ml}$ cup of roast and ground coffee might supply from $20 \mathrm{mg}$ 5-CQA (weak brew, very dark roast) up to $675 \mathrm{mg}$ 5-CQA (strong brew, very pale roast robusta). Soluble coffee powder ( $2 \mathrm{~g}$ per cup) has been reported to include 50-150 mg of 5-CQA (Clifford 1999).

Yerba mate, a traditional South American beverage prepared from the leaves of Ilex paraguariensis A. St.Hil., is rich in mono- and diCQAs. There have been few investigations of the brew composition. However, it was proved that green mate material bought and brewed in Europe provided 107-133 mg CQAs per approx. $200 \mathrm{ml}$, of which diCQA represented onethird. In contrast, roasted mate provided $16-41 \mathrm{mg}$ CQAs per $200 \mathrm{ml}$ (Clifford and Ramirez-Martinez 1990). Mazzafera (1997) reported that the traditional manner of brewing the authentic material (50-60 g of leaves per liter of boiling water) provided the isolation of 480-520 mg of 5-CQA.

In view of the above presented examples showing the CQAs content in plants, it can be stated that among all CQAs, 5-CQA is the most ubiquitous compound that is present in larger quantities. For these reasons 5-CQA is commonly employed as a quality marker in the control of various natural products and it belongs, undoubtedly, to the most frequently studied representatives of the chlorogenic acids family. The other reasons for researchers interest in 5-CQA include: its important role in the plant response to stress, is an important biosynthetic intermediate, for example, in lignin biosynthesis, and exhibits antiviral, antibacterial, antifungal and anticarcinogenic effects combined with a relatively low toxicity and side effects (Johnston et al. 2003). The other compound that attracts the researcher's attention, due to its biological properties, is 1,3-diCQA. This compound, in contrast to 5-CQA is mainly characteristic of the Asteraceae family especially of artichoke. Yet despite a rare occurrence and a low content in plants, 1,3-diCQA is the best known and frequently investigated derivative of dicaffeoylquinic acids (Lattanzio et al. 2009). 1,5-diCQA and 3,4-diCQA, though they occur in larger quantities in many plants, they are not so intensively investigated by researchers (Lattanzio et al. 2005, 2009; Upadhyay and Rao 2013). 


\section{The beginnings of CQAs chemistry}

As mentioned in Upadhyay and Rao (2013) the first report on chlorogenic acids, concerning the properties of chlorogenic acid alone, appeared most likely in 1837. The first documented reference, however, comes from 1844. In that year Rochleder noticed that caffeine in green coffee beans could combine with an acid that could be precipitated with lead salts. The free acid, generated by the treatment of precipitate with sulfuric acid, gave a yellow colour on the addition of ammonia. Two years later the same researcher proposed an empirical formula of $\mathrm{C}_{16} \mathrm{H}_{9} \mathrm{O}_{8}$ for the free acid and reported that the yellow ammonical solution became green on exposure to oxygen. (That is why despite the absence of chlorine the compound is called "chloro".) Later that year Payen announced the isolation of a crystalline potassium caffeine chloroginate that formed about $3.5 \%$ of green coffee beans and proposed an empirical formula of $\mathrm{C}_{14} \mathrm{H}_{8} \mathrm{O}_{7}$. Gorter reported the melting point at $206-207{ }^{\circ} \mathrm{C}$ for pure white crystals of chlorogenic acid and proposed an empirical formula of $\mathrm{C}_{32} \mathrm{H}_{38} \mathrm{O}_{19}$. Alkaline hydrolysis at low temperatures yielded caffeic acid (CA) and quinic acid (QA) in equimolar quantities. To make this observation compatible with the proposed empirical formula, Gorter suggested that CA associates with QA to produce hemi-CQA, two molecules of which condensed to give chlorogenic acid. In 1920 Freudenberg reported that the enzyme tannase hydrolyzes chlorogenic acid to the equimolar quantities of CA acid and QA. In 1932 Fischer and Dangschat deduced that chlorogenic acid is 3-caffeoylquinic acid (Upadhyay and Rao 2013). (According to a pre-IUPAC nomenclature for cyclitols, the positional number of carbon atoms in the quinic acid ring were assigned in an anticlockwise manner.) Under the current IUPAC recommendations (positional number assigned to the carbon atoms of the quinic acid ring in the clockwise sense), 3-caffeoylquinic acid is now designated 5-Ocaffeoylquinic acid (5-CQA, see Fig. 1).

The first report on the dicaffeoylquinic acid derivatives was prepared by Panizzi and Scarpati (1954) and concerned the isolation of cynarin from artichoke leaves. In this report, cynarin was characterized as a yellowish-coloured crystalline substance exhibiting left-handed rotatory power and a weak acid reaction that is slightly stable in the air alkaline solution and changes to green in the presence of ferric chloride. The cynarin structure was initially described as 1,4-O-dicaffeoylquinic acid. Later the compound was identified as 1,5-O-dicaffeoylquinic acid by the same researchers. At present, according to the IUPAC recommendation cynarin is called 1,3-O-dicaffeoylquinic acid (see Fig. 1).

\section{CQAs properties}

Compounds belonging to the chlorogenic acid family exhibit very differentiated physicochemical properties dependent on the identity, number and position of the acyl residues esterified with the quinic acid as well as on the functional groups present on the aromatic moiety of the acyl residues.

The monoCQAs, in general, and the less polar diCQAs, in particular, are more soluble in the lower alcohols or alcohol-water mixtures. They are insoluble in benzene, chloroform, and petroleum ether. The diCQAs, in addition to the lower alcohols, are well dissolved in ethyl acetate, butyl acetate and acetone. Taking into account the position of acyl residue in the quinic acid, water solubility declines in the sequence: 1- > 3- > 4- > 5- (Upadhyay and Rao 2013). Though the frequently observed elution order of monoCQAs in the reversed-phase liquid chromatography is as follows: 1-, 3-, 5- and finally 4- (Schutz et al. 2004). For diCQAs the order can be presented as follows: 1,3$>1,4->3,4->1,5->3,5->4,5-\quad$ (Gouveia and Castilho 2009). In general, those CQAs with a greater number of free equatorial hydroxyl groups in the quinic acid residue are more water soluble than those with a greater number of free axial hydroxyl groups (Clifford et al. 2005). As for the influence of functional groups present on the aromatic moiety of the acyl residues, a hydroxyl group raises the polarity whereas a methoxy one reduces it. As regards the effects of the identity of hydroxycinnamic acid derivatives, water solubility declines in the following order: 5-CQA > 5CoQA > 5-FQA (Upadhyay and Rao 2013). Considering the effect of geometrical isomerism on CQAs hydrophobicity, according to Clifford et al. (2008) the cis-5-acyl isomers are more hydrophobic than their counterparts whereas for the 4-acyl and 3-acyl CQAs the reverse is true.

In the case of CQAs one of the characteristic processes responsible for modifying the structure of CQAs is acyl migration for the first time reported by 
Hanson 1965), and nowadays widely discussed in the literature (Deshpande et al. 2014; Li et al. 2015). This process describes the migration of cinnamoyl moieties from one quinic acid alcohol group to another. It is responsible for regioisomerization of appropriate CQAs, thus it constitutes a special case of transesterification reaction. The migration process is temperature and $\mathrm{pH}$ dependent with the increased transesterification taking place at basic $\mathrm{pH}$ (Wianowska et al. 2015b; Dawidowicz and Typek 2010; Deshpande et al. 2014). Moreover, the process can be intra- and/or intermolecular, and the latter can simultaneously compete with intramolecular acyl migration as well as hydrolysis. According to Deshpande et al. (2014) under the aqueous basic conditions the intramolecular acyl migration in monoCQAs is fully reversible. Through intramolecular acyl migration in monoCQAs, 5-CQA first isomerizes to 4-CQA (also known as cryptochlorogenic acid), then to 3-CQA (neochlorogenic acid), and finally to 1-CQA (Wianowska et al. 2015b; Dawidowicz and Typek 2010; Xue et al. 2016). It should be added that among the all 5-CQA isomers the presence of 3- and 4-CQA was confirmed in plants while the 1-CQA isomer is known only from theoretical considerations (Clifford 2000). Of the three naturally occurring CQAs i.e. 5-, 4- and $3-$, the latter is the least stable and decomposes to quinic and caffeic acids even before the acyl migration takes place. Similarly to the monoCQAs, the diCQAs could also undergo the intramolecular acyl migration. Yet the monoCQAs are generally much more stable than the diCQAs under the same conditions. Taking into account the diCQAs stability, in Xue et al. (2016) it was reported that 4,5-diCQa is much more stable than 3,4-diCQA and 3,5-diCQA. Citing the authors of this report, this might be due to the fact that diCQAs are more stable when the ester bond is attached to the quinic acid exists as an equatorial bond rather than the axial one. Only one ester bond of 3,4diCQA and 3,5-diCQA exists as the equatorial bond while all ester bonds of 4,5-diCQA exist as the equatorial ones.

Apart from the acyl migration process, researchers reported other chemical changes for CQAs, such as hydrolysis of the ester bond, decarboxylation of cinnamoyl moieties, epimerization at the quinic acid, dehydration, lactones formation, and reaction with water or methanol/ethanol (Clifford 2000; Dawidowicz and Typek 2010; Deshpande et al. 2014;
Wianowska et al. 2015a, b). It should be added that the above mentioned processes can compete with each other, as in the case of hydrolysis and acyl migration competition under aqueous basic conditions (Deshpande et al. 2014).

CQAs are highly susceptible to the influence of temperature. As temperature increases they are not only more readily subjected to intramolecular isomerization and transesterification but also to degradation (Farah et al. 2005; Jaiswal et al. 2012; Wianowska et al. 2015a, b; Dawidowicz and Typek 2010, 2017; Xue et al. 2016). The diCQAs degrade to the corresponding monoCQAs and then to caffeic and quinic acids. It should be added that the latter compounds i.e. caffeic and quinic acids are also products of monoCQAs degradation. The number of derivatives and the amount of each formed compound depend on the heating time and temperature (Farah et al. 2005; Jaiswal et al. 2012; Wianowska et al. 2015b; Dawidowicz and Typek 2010, 2017). It should be stressed, however, that this evaluation strongly depends on the sensitivity of the applied analytical instrument. According to Xue et al. (2016), the total of eight degradation products of 3,4-diCQA, six degradation products of 3,5-diCQA, four degradation products of 1,3-diCQA, and three degradation products of 4,5-diCQA were identified in the diCQAs solutions. In the case of monoCQAs solutions, only two degradation products of 3-CQA, three degradation products of 5-CQA, and four degradation products of 4-CQA were detected and identified (Xue et al. 2016). However, as demonstrated in Dawidowicz and Typek (2015), long heating of the 5-CQA solution alone produces at least twenty compounds being 5-CQA derivatives as well as its reaction product with water and methanol.

Due to the 1,2-disubstituted alkenic molecular structure, CQAs are photosensitive and exposed to ultraviolet or visible light they undergo trans-cis isomerization. Isomerization kinetics is affected by irradiation time, wavelength and temperature. Yet for the samples stored at room temperature in methanol, significant degradation of monoCQAs was observed after 7 days of their exposition to light (Xue et al. 2016). This can indicate that a single factor such as temperature, light or solvent has an insignificant effect on the stabilities of monoCQAs. However, under dual factors of light and temperature both mono- and diCQAs decompose readily (Dawidowicz and Typek 
2010; Deshpande et al. 2014; Xue et al. 2016). The study of CQAs stability in two solvents (methanol and aqueous methanol solution, 50\% v/v) kept in brown glass bottles at $4{ }^{\circ} \mathrm{C}$, described in (Xue et al. 2016), showed slow degradation of CQAs. Meanwhile, noticeable drop in the concentrations of CQAs was observed when kept in a transparent glass vessel at room temperature. CQAs degraded in methanol to the respective adducts or esters of methanol.

\section{Pro-health biological activity of CQAs}

There are many advantageous health properties associated to the family of CQAs such as hepatoprotective and choleretic activities, antioxidant, antiviral, antibacterial, anti-cancer and anti-inflammantory properties, modulation of gene expression of antioxidant enzymes, reduction of the risk of cardiovascular disease via suppressing $\mathrm{P}$-selectin expression on platelets, and reduction of the relative risk of diabetes type 2 and Alzhaimer's disease (Kwon et al. 2000; Johnston et al. 2003; Shearer et al. 2003; Ludwig et al. 2014). Some of these properties are already well known and tested, others have not been proven yet and are now under close scrutiny. This is, for example, the case of the assumption that CQAs can be helpful in fighting obesity and modulate glucose-6-phosphatase involved in the glucose metabolism (Shearer et al. 2003), and that they are a novel class of antiviral compounds, namely inhibitors of integrase, being potent inhibitors of HIV-1 replication, both in vitro and in vivo (Kwon et al. 2000). The pro-health properties are attributed to trans isomers of CQAs. However, in the light of more and more frequent reports on the presence of cis isomers in plants, the most recent research concerns the determination of the biological significance of $c i$ isomers relative to their trans counterparts. For instance, in Chen et al. (2011) it was proved that antituberculosis activity of cis cinnamic acid is approximately 120 -fold stronger than the trans form.

Finally, it should be emphasized that despite the abundance of the data demonstrating CQAs biological activities, some of them are still controversial. This is the case with their antioxidant properties. CQAs have been reported to inhibit the formation of reactive oxygen species in vitro and act as their scavengers. Thus they can play a beneficial role in the prevention of oxidative and age-related diseases (Kweon et al. 2001; Ludwig et al. 2014). According to new results, however, the issue whether these compounds are potent anti-oxidants in vivo, it remains debatable. In the opinion of (Upadhyay and Rao 2013) this is a consequence of the fact that CQAs properties switch from anti- to pro-oxidant activity, depending on their concentration, the presence of free transition metal ions, or on their redox status. Yet human studies on the bioavailability of chlorogenic acids in coffee, as the dominant source of CQAs, show that the absorption of CQAs is low and their half-life in plasma is short (Clifford et al. 2017; El-Seedi et al. 2012). Therefore the capabilities of dietary CQAs to act as scavengers of free radicals in plasma are limited. In addition to being poorly absorbed, CQAs are also extensively metabolized to forms that are less potent antioxidants. In Galati and O'Brien (2004) and Lotito and Frei (2006) it was even stated that the pro-health properties of CQAs do not result from their antioxidant properties but from their toxicity which triggers the physiological production of the actually antioxidant uric acid.

In the view of the CQAs biological properties and their high susceptibility to the negative impact of surroundings (solvent type, $\mathrm{pH}$, temperature and even light), it is obvious why so many attempts have been undertaken to modify the way in which the compounds are isolated. Searching for new and alternative methodologies of CQAs extraction from plants is additionally supported by the fact that some of those compounds can be found in nature at very low concentrations. Thus to obtain their sufficient amounts very effective isolation methods are needed, the more that their structural diversity and complexity make chemical synthesis rather unprofitable.

\section{Isolation of caffeoylquinic acids}

The common procedures used so far for determination of compounds in plants and related products involve two-step processes. In the first one, the compounds are liberated from the pre-prepared sample matrix. In the second, they are analysed using chromatographic techniques more frequently. Depending on the sample matrix type, different procedures are applied for sample pre-treatment. Solid samples, such as plants and solid food stuffs, are usually homogenized which can be preceded by lyophilisation or freezing with 
liquid nitrogen. Liquid samples, such as beverages, are usually first filtered and/or centrifuged, and then they are subjected to direct analysis or, what is more common to enhance the determination selectivity to further steps of preparation.

To isolate fully the compounds released from the matrices and/or to concentrate them, a broad range of sample preparation techniques (e.g. distillation, sublimation, extraction to gas, liquid or solid phase) is currently being used. Most approaches to the plant components analysis involve the application of liquid extraction methods, such as extraction in the Soxhlet apparatus, under reflux and maceration. These are the so-called conventional liquid extraction techniques. Their extraction ability is based on the extracting power of different solvents and the application of heat and/or mixing. The conventional techniques are known for many disadvantages, such as being timeconsuming, relatively high solvent consumption, often unsatisfactory reproducibility and poor extraction of polar substances. In an attempt to eliminate the drawbacks of the conventional liquid extraction and to enhance the extraction process and, make it more environmentally friendly, the innovative extraction methods such as microwave-, ultrasound-, pressure-, and enzyme- assisted extraction together with supercritical fluid extraction have been developed and introduced. Their applicability for the isolation of various plant constituents, including CQAs, both on a small (laboratory) and large (manufactering) scale, is increasingly growing (Kaufmann and Christen 2002; Waksmundzka-Hajnos et al. 2004; Li et al. 2005; Carabias-Martinez et al. 2005; Dawidowicz and Wianowska 2005a, b; Dawidowicz et al. 2009; Wianowska et al. 2009, 2014a, b, 2015b; Mustafa and Turner 2011; Upadhyay et al. 2012; Dent et al. 2015; Wianowska and Wiśniewski 2015; Tena et al. 2015; Wianowska and Dawidowicz 2016a, b; Srinath and Maheswari 2016). This is understandable as these techniques, by limiting the use of mostly toxic organic solvents could improve extracts quality additionally, which is important taking into account the CQAs applicability as pro-health additives to foods and supplements. On the other hand, to investigate the composition of plants in the proper way the matrix solid-phase dispersion technique (MSPD) and its variant, the sea sand disruption method (SSDM), are increasingly recommended (Dawidowicz and Wianowska 2009; Dawidowicz et al. 2013; Wianowska et al. 2015a, 2017; Wianowska 2015; Wianowska and Dawidowicz 2016a, b). This is due to the fact that they do not induce any transformation and/or degradation processes in the analysed substances and, in consequence, they allow to establish a true concentration of individual CQAs in the examined plants and to determine which chlorogenic acids derivatives are native plant components and what their concentration level is. In other words, the application of MSPD/ SSDM in plant analysis allows to eliminate errors that may arise or might have arisen in the study of chlorogenic acids and their derivatives in plant metabolism.

Ultrasound assisted solvent extraction

The mechanism of ultrasounds in liquids relies in the mechanical effect caused by the implosion of cavitation bubbles generating macro-turbulence, high-velocity inter-particle collisions and perturbation in micro-porous particles of the natural materials which accelerates diffusion and enhances the mass transfer (Srinath and Maheswari 2016). Due to the possibility of using these phenomena to reduce extraction time and increase yield of heat-sensitive compounds at lower processing temperatures, the increasing interests in application of ultrasounds to plants and plant products extraction have been observed (Dent et al. 2015). In addition, the ultrasound-assisted solvent extraction (UASE) technique provides the greater opportunity of enhancing its extraction ability. Apart from the careful choice of an appropriate solvent characteristic of the conventional techniques, the process can be further optimized and this is an important part of the UASE process. During the optimization ultrasounds frequency, their amplitude, number of the applied extraction cycles, irradiation time and nominal output power are taken into consideration. It is worth noting that in addition to the low overall process temperature, the use of highfrequency sonication also reduces the degradation of compounds extracted from plants. Another advantageous feature of UASE, that is equally important, is the cost of equipment necessary for the technique. The cost is low as the process is most often performed in ultrasonic baths being in each laboratory. Yet the process can be realized in a different way applying an ultrasonic disintegrator. In this case the ultrasound emitting tip is directly immersed in the extraction 
mixture and the process is called the focused ultrasound assisted solvent extraction (FUASE) (Tena et al. 2015). FUASE, compared to UASE, by some researchers is considered to be even more reproducible and faster mainly due to a few times greater ultrasonic power (Martinez-Moral and Tena 2011). It should be emphasized, however, that regardless of the extraction way, both variants can generate reactive oxygen species that they can cause degradation of some compounds and their lower concentrations in extracts if the sonication is continued over a long period. Hence, an important issue when using ultrasound for plants extraction is applying water/alcohol mixtures as extractants (Vinatoru et al. 2017). This reduces the generation of oxidising species from the decomposition of water, because alcohols are much more stable in terms of homolytic cleavage. The validity of this statement is confirmed by the following two examples documenting the relatively fast and effective isolation of 5-CQA from various matrices at room temperature.

Li et al. (2005) investigated an ultrasound-assisted method for the extraction of 5-CQA from fresh leaves of du-zhong (Eucommia ulmoides Oliv.). During the experiments the influence of four extraction variables (solvent type, its volume and concentration as well as extraction time) on the yield of 5-CQA was examined. As solvents water, methanol, ethanol and isopropanol were tested and it was found that the mean extraction efficiency of 5-CQA decreases in the order: methanol $>$ ethanol $>$ water $>$ isopropanol. Adding water to methanol $(70 \%$ methanol in water, $v / v)$ additionally increases the 5-CQA yield. An increase of extraction efficiency was also observed with the increase of the ratio of solvent volume to the sample (the ratio of 20 was optimal) and the number of extraction cycles. It was proved that, in comparison to the conventional extraction techniques, triplicate extraction of 5-CQA for 30 min using a fresh extractant each time reveals the highest 5-CQA yield. The optimized UASE process was applied by the researchers for the extraction of 5-CQA from bark of $E$. ulmodies and four other traditional Chinese herbs and found that independently of the plant matrix type the isolation efficiency of UASE is very high and the obtained results highly reproducible.

Saleh et al. (2016) studied the application of UASE for the extraction of 5-CQA from artichoke leaves. The extraction was performed by using $80 \%$ methanol at room temperature for $15 \mathrm{~min}$. The results showed that under the UASE conditions used, a significant increase in 5-CQA yield (up to 50\%) was obtained compared to maceration at room temperature. In this light, the advantages of using UASE to isolate CQAs from plants, also on an industrial scale, are clear. Not only the processing time is shorter at much lower costs, but also the extraction efficiency is higher due to preventing the thermal degradation of the compounds and guaranteeing the greater contact area between solid and liquid phase, compared with the traditional extraction methods.

\section{Microwave assisted solvent extraction}

The microwave assisted solvent extraction (MASE) is considered as a novel and alternative method for isolation of CQAs from plants and related products using microwave heating. The principle of microwave heating, conversely to the conventional heating, is based on direct absorption of microwave energy and its conversion into thermal energy when the microwaves are passed through the medium (Eskilsson and Bjorklund 2000; Kaufmann and Christen 2002). The conversion of electromagnetic energy to heat occurs via two mechanisms, specifically, by dipole rotation and ionic conduction, i.e. by means of reversal of dipoles and displacement of charged ions present in both the sample and the solvent. These two mechanisms usually occur simultaneously which converts microwave energy into thermal one effectively. Dipole rotation takes place when the dipolar molecules attempt to follow the electric field in the same alignment. The induced oscillation leads to collisions between the dipoles and the surrounding molecules and thus creates heat. Ionic conduction refers to the migration of ions under the influence of the electric field produced by microwaves. In this case heat is generated due to the resistance of the medium to flow ions (Eskilsson and Bjorklund 2000). It should be added that in both mechanisms the generated thermal energy is immediately redistributed in the medium, which leads to homogeneous heating of the medium.

The MASE effectivity can be affected by a large variety of factors, such as frequency and power of microwaves, duration of microwaves irradiation, moisture content and particle size of plant samples, type and concentration of solvent, ratio of solid to liquid, extraction temperature and number of 
extraction cycles. Of these factors, solvent is regarded to be one of the most important parameters, which affects not only the solubility of compounds but also the absorption of microwaves energy. The higher the dielectric constant and dielectric loss, the higher is the solvent capacity to absorb microwave energy and the faster solvent heating. It should be added, however, that both polar and non-polar solvents can be used in MASE. Moreover, by combining solvents differing in their dielectric constant, the extractant properties and its selectivity can be modified. Nevertheless, the amount of water content in the extracted material and/or in solvent significantly influences the extraction yield. Therefore, the isolation of the compounds from plants can be carried out without the need for the use of organic extractants, in the so-called solventless mode. Other advantages of MASE are improved extraction efficiency and shortened extraction time, resulting in decreased costs and environmental impact. Due to these strong benefits, MASE is considered as a good alternative to traditional extraction processes of plant metabolites. A serious concern that might stand to be true when using MASE for CQAs isolation can be explained by the fact that the amount of microwave energy generated in the system can be sometimes so hight that it easily can cause a loss of thermally unstable compounds and consequently a decrease in their extraction efficiency. The following examples show, however, that the skillfully conducted CQAs extraction process by MASE using the appropriate volume of water in several short extraction cycles can give a higher CQAs yield compared to the conventional extraction techniques.

The MASE applicability for CQAs extraction from green coffee beans was described in Upadhyay et al. (2012). During the investigations the influence of four extraction variables (solvent type, extraction time, extraction temperature and microwave power) on the extraction efficiency of 5-CQA was tested. Among the examined solvents (ethanol, methanol and water), water proved to be the best extractant giving the highest yield of 5-CQA. This is due to the fact that the dielectric constant and polarity of water are higher than those of the alcohols. It was observed that extending the extraction time from 2 to 5 min resulted in the increased extraction efficiency. However further extension of the time resulted in reduction of 5-CQA yield. Taking into account the effect of extraction temperature on the total CQAs yield, it was shown that the highest yield was obtained at the temperature of $50{ }^{\circ} \mathrm{C}$. Finally, testing the effect of microwave power it was noticed that the total CQAs yield was increased with the increase the microwave power up to $800 \mathrm{~W}$. Comparing the MASE results with those of the conventional extraction techniques, the authors concluded that the optimized MASE procedure isolates 5-CQA equally effectively as the conventional techniques. At the same time the authors stressed that the application of MASE reduced both time and solvent consumption. Therefore MASE can be recognized as a more environmentally friendly process providing high quality extracts rich in CQAs.

According to some researchers, the performance of MASE is highly influenced by the solid to liquid ratio. Generally, a larger solvent volume can dissolve compounds of interest more effectively and lead to a higher extraction yield. As shown in (Li et al. 2004) the extraction yield of 5-CQA from Eucommia ulmodies Oliv. increased with the improvement of the solvent volume (i.e. the reduction of the solid to liquid ratio). The 5-CQA yield increased steadily when the plant mass was kept constant and the liquid volume was raised. That is to say, the extraction yield of plant constituents depends on how the solid to liquid ratio is regulated. Therefore in MASE the plant sample extraction is typically conducted in multiple steps, using the consecutive extraction cycles with a fresh portion of the extractant. This practice helps to improve the extraction yield and prevents prolonged heating of the sample in the same solvent volume. In addition, the use of fresh portions of extractant prevents the saturation of the solvent with analytes, increasing their mass transfer and extraction kinetics (Routray and Orsat 2012). In other words, MASE can extract compounds more rapidly, thus the heat-sensitive compounds are shortly exposed to potentially harmful conditions and in consequence, their yields can be higher in comparison to those obtained by the conventional extraction methods.

\section{Pressurized liquid extraction}

Pressurized liquid extraction (PLE) is known under different names, such as accelerated solvent extraction (ASE), pressurized solvent extraction (PSE), enhanced solvent extraction (ESE), pressurized hot water extraction (PHWE), subcritical water extraction or superheated water extraction (SWE). The technique 
has gained popularity due to its lower organic solvents consumption, and even their complete elimination, and significantly shorter extraction times required for full isolation of bioactive compounds from plants. These are the reasons explaining also why it is described as a green extraction technique (Mustafa and Turner 2011). In PLE a sample enclosed in the stainless steel extraction vessel is extracted in an inert atmosphere with a solvent at high pressure and at a temperature exceeding the boiling point of the solvent under the atmospheric pressure. Due to the increase of solubility and mass transfer and, also decrease of viscosity and surface tension of solvents, higher extraction yields compared to the conventional extraction methods are observed. The technique has proven to be an equivalent or superior alternative to the extraction of different analytes from various matrices (Carabias-Martinez et al. 2005; Dawidowicz and Wianowska 2005a, b; Dawidowicz et al. 2009; Wianowska et al. 2009; Mustafa and Turner 2011; Wianowska and Wiśniewski 2015). For isolation of polar compounds PLE is also considered a potential technique alternative to the supercritical fluid extraction (Kaufmann and Christen 2002).

Alonso-Salces et al. (2001) were the first to apply PLE for isolation of plant constituents. The researchers optimized the PLE process for extraction of phenolics in the Golden Delicious apple peel and pulp taking into account the effects of experimental variables such as solvent composition (water, methanol:water 20:80 v/v, methanol:water 50:50 v/v, methanol:water 70:30 v/v, methanol), extraction temperature $\left(40{ }^{\circ} \mathrm{C}, 60{ }^{\circ} \mathrm{C}, 80^{\circ} \mathrm{C}, 100{ }^{\circ} \mathrm{C}\right)$, extraction time (5, 10 and $15 \mathrm{~min})$ and pressure (1000, 1250 and 1500 p.s.i.), and proved that the most powerful extraction mixture was $60 \%$ solution of methanol in water at $60{ }^{\circ} \mathrm{C}$. No significant changes in the extraction efficiency of compounds were found studying the effect of extraction time and pressure. However, according to Erdogan et al. (2011) for quantitative 5-CQA isolation from various parts of Anatolia propolis under PLE conditions using the ethanol:water: $\mathrm{HCl}$ extractant mixture $(70: 25: 5, \mathrm{v} / \mathrm{v} / \mathrm{v})$ at $40{ }^{\circ} \mathrm{C}$, the extraction time should be extended up to $15 \mathrm{~min}$ and the pressure raised to $1500 \mathrm{psi}$. This observation is consistent with the findings of other researchers (Lou et al. 1997; Dawidowicz and Wianowska 2005a, b, 2009) who independently observed that the PLE efficiency is pressure- and time-dependent when the extraction process takes place at lower temperatures (below the boiling point of the solvent) and the plant material swells easily. In other cases, the pressure does not affect the extraction efficiency because the purpose of pressurizing the extraction vessel is to prevent the solvent from boiling at the extraction temperature and to ensure that the solvent remains in an intimate contact with the sample. Luthria (2008) showed that not only the type of plant matrix but also the size of plant particles, the solid-toliquid ratio, and especially the volume of extractant used to flush the extraction vessel after completion of the process (so-called the flush volume) affects the extraction yield of phenolics from the parsley flakes Petroselinum crispum Fuss.).

One of the characteristics of PLE is to promote the use of water as an extractant of less polar bioactive compounds. The efficiency of this approach is mainly affected by temperature as the polarity of water under pressure changes with temperature. At lower temperatures the water polarity is high but with the temperature increase it decreases so that the polarity of pressurized water resembles the polarity of polar organic liquids. Due to the elimination of the use of organic solvents, this approach seems to be milder compared to conventional techniques, avoiding the production of artifacts. A more thorough analysis of the application of water under the PLE conditions for extraction of natural compounds reveals, however, that this approach is not free from drawbacks. In Wianowska et al. (2015b) it was prove that 5-CQA isomerizes to 3- and 4-CQA and undergoes transesterification, hydrolysis, and reaction with water even using the fast PLE. In Dawidowicz and Typek (2010) it was found that heating of the 5-CQA water solution in the PLE apparatus leads even to formation of 4,5dicaffeoylquinic acid and the presence of other isomers of dicaffeoylquinic acids cannot be excluded. These examples indicate the need for a thorough qualitative and quantitative study of the composition of natural extracts not only at the final stage of extract production, but also at the initial stages of determining optimal extraction conditions. This is important because due to the proven susceptibility of CQAs to the negative effects of high temperature, light, $\mathrm{pH}$ and the extractant type, it is not possible to find the PLE conditions under which the transformation of 5-CQA would be completely imposible. The selection of optimal extraction conditions should be a compromise 
in conditions that guarantee the highest 5-CQA yield and the lowest amount of 5-CQA derivatives. It seems that the best choice will be the use of a short-lasting PLE process, giving a lower number and quantity of CQA derivatives.

\section{Supercritical fluid extraction}

At present, supercritical fluid extraction (SFE) is increasingly used in numerous industries applications including food (decaffeination of coffee), pharmaceutical (extraction of pharmacologically active substances), cosmetics (obtaining essential oils) and fuel. This is due to the fact that the obtained extracts are free from all inorganic compounds. The relatively low temperature of the process does not cause degradation of proteins, vitamins and other active ingredients of the plant and thus does not reduce their health and organoleptic quality. In addition, the entire process runs without air, the product obtained has no chance of undergoing any oxidative changes.

SFE has several advantages over the conventional extraction techniques: it is a flexible process due to the possibility of adjustment of the solvent power or selectivity of the supercritical fluid (SCF), it allows protection of polluting organic solvents, and it eliminates the expensive cost of postprocessing of the extract for solvent reduction. SCF of any substance is present where the temperature and pressure are above its critical point, forming a homogenous phase with both liquid-like and gas-like properties. Due to its gaslike low viscosity and high diffusivity, a SCF, when used as a solvent, can easily penetrate plant materials with a rapid mass transfer rate. In addition, the density of a SCF can be altered by adjusting the pressure and temperature, hence SCF density is said to be tunable. The most commonly used solvent in SFE is carbon dioxide $\left(\mathrm{CO}_{2}\right)$ in its supercritical fluid state, due to the following factors: it is readily available, it is a reusable gas, and it has a low critical temperature of $31.1{ }^{\circ} \mathrm{C}$ and relatively low critical pressure of 72.8 bar (Khaw et al. 2017).

SFE, due to the chemical character of superctricial $\mathrm{CO}_{2}$, is a technique commonly used to extract nonpolar pharmacologically active compounds from plants. To isolate polar compounds, the SCF should be enriched with a suitable polar solvent (so-called the co-solvent). The co-solvent effect is associated with the modification of intermolecular interactions forces and the increase in the density of extractants. Azevedo et al. (2008) showed that the addition of ethanol and isopropyl alcohol as co-solvents increases the yield of CQAs from green coffee beans. Experiments of Machmudah et al. (2011) revealed that not only the addition of a polar solvent but also the way of conducting the extraction affects the yield. These researchers proved that the counter-current extraction in flow mode is more effective compared to both batch and co-current extraction with $\mathrm{CO}_{2}$. In $\mathrm{Wu}$ et al. (2015), 5-CQA was extracted from flower buds of Lonicera japonica Thunb by using modified supercritical $\mathrm{CO}_{2}$ extraction. The highest yield of 5-CQA was obtained for the following conditions: $32 \%$ ethanol in water (v/v), temperature of $74{ }^{\circ} \mathrm{C}$ and pressure of 289 bar. This study showed that as far as the change in ethanol concentration and extraction pressure can significantly affect the recovery of chlorogenic acid, the temperature effect is negligible. Chlorogenic acids are thermally unstable compounds, so the lack of temperature influence on the SFE process efficiency makes this method an attractive alternative to the conventional extraction techniques.

\section{Enzymatic extraction}

In the last few years, enzymatic extraction has gained research interest due to the fact that the method is completely ecologicall and the obtained extract is of high quality that allows it to be used for the production of medicines, dietary supplements and specialized cosmetics. The basic principle of this method is that the enzymes hydrolyze the plant cell wall and disrupt it completely under optimum experimental conditions, to release components. Yet, this method in not free from drawbacks and the main problem is the selection of an appropriate enzyme that will enable the extraction of the desired compound. In addition, the activity of enzymes is strictly dependent on the temperature and $\mathrm{pH}$ of the environment, thus these parameters must be constantly monitored during the process. As an example of the use of enzymatic process for the isolation of CQAs from natural materials, the work of Torres-Mancera et al. (2013) can be given. The researchers proved that after a few hours stiring of coffee pulp with enzymes (in darkness, at $40{ }^{\circ} \mathrm{C}$ ) chlorogenic acid can be effecivelly separated from the waste material using a small amount of ethyl acetate. 
Matrix solid phase dispersion

As it was stated before for faster CQAs isolation from plants, temperature is usually applied. Yet as it was proved in (Wianowska et al. 2015b) even in the shortlasting PLE process 5-CQA undergoes degradation and transformation to other compounds which can be mistakenly treated as new plant components or can lead to erroneous quantitative estimation of plant composition. Thus the challenge in the CQAs analysis in plants is to obtain an extract whose composition would exactly reflect the true composition and concentrations of CQAs, so important for researchers studying plant metabolism. Taking into account the fact that temperature is the main factor responsible for CQAs degradation, it would be more advantageous to use the extraction techniques at ambient temperature. According to Dawidowicz and Wianowska (2009), Wianowska et al. (2015a, 2017), Wianowska (2015) and Wianowska and Dawidowicz (2016a, b) one of such techniques can be the matrix solid-phase dispersion (MSPD). In addition, it is very simple, quick and cheap. More importantly, it proved to isolate effectively different compounds from various plant matrices.

MSPD consist in direct mechanical blending of biological sample with the solid material i.e. octadecyl modified silica (C18) which helps to disrupt plant cells. After a few minutes of vigorous homogenization, the crushed plant material is transferred into a SPE tube and the released plant constituents are eluted with an appropriate eluent. Instead of C18 sorbent, sand can be applied in the MSPD process. In this case the method is called the sea sand disruption method (SSDM). There are many examples of procedures in which this method is successfully applied for release of various compounds from different plant matrices, including CQAs (Teixeira and Da Costa 2005; Teixeira et al. 2006; Dawidowicz and Wianowska 2009; Dawidowicz et al. 2011, 2013; Oniszczuk et al. 2014; Wianowska 2015; Wianowska et al. 2015a, 2017; Wianowska and Dawidowicz 2016a, b). In addition, there are the examples proving that the amounts of compounds released by this method are higher than those revealed by the conventional approaches, although the reasons explaining this fact given by the authors are different.

Teixeira et al. (2006) compared the efficacy of SSDM and MSPD as well as the conventional solid- liquid extraction method for isolation of phenolics from the fig tree (Ficus carica L.) leaves and proved that both SSDM and MSPD disclose higher extraction efficiency. According to the authors, higher MSPD and SSDM yields are due to better sample destruction which, by exposing the plant cell components to a solvent, tend to yield richer extracts. Applying MSPD and PLE for the isolation of phenolics including CQAs from the field horsetail (Equisetum arvense L.) Oniszczuk et al. (2014) indicated the lack of temperature-induced degradation of compounds as the reason for higher MSPD efficiency. This statement, however, was not supported by experimental proves. In Wianowska et al. (2015a) comparing the CQAs derivatives obtained from the 5-CQA standard with those revealed under the identical conditions for different plants [black elder (Sambucus nigra L.) and hawthorn (Crataegus L.) flowers, nettle (Urtica dioica L.) and yerba maté (Ilex paraguariensis A.St.-Hil.) leaves; St John's wort (Hypericum perforatum L.) herb and green coffee beans] by means of extraction under reflux and SSDM in different variants, it was unambiguously demonstrated that the SSDM method does not induce any transformation and/or degradation processes in CQAs.

Finally, it is worth mentioning that in addition to the C18 sorbent and sand, other materials are tested as dispersive agents. In Xu et al. (2016), to extract CQAs isomers from different honeysuckle samples a novel $\beta$ cyclodextrin based matrix solid phase dispersion method was developed. The results proved that the proposed method provides good precision and high recoveries of monoCQAs as well as diCQAs. Moreover, according to the authors, the method possesses many practical advantages, including rapidity, high sensitivity and less consumption of samples and organic solvents.

\section{Centrifugal partition chromatography}

One of the typical problems of CQAs quantitative analysis in plants and plant products is the very limited number of commercially available standards of these compounds. Moreover, some commercial standards are incorrectly described at a regio-isomer level (Clifford et al. 2017). Therefore, to overcome this problem without compromising the quality of the results, two approaches are typically used. In the first one the use of surrogate standards such as a green coffe 
extract or artichoke extracts characterized by LC-MS fragmentaion and relative retention times is considered. In the second, the compounds are isolated from plant extracts by different chromatographic procedures, and then subjected to meticulous purification.

Romero-Gonzalez and Verpoorte (2009) studied the application of centrifugal partition chromatography with gradual reduction of the ionic strength of the mobile phase in order to separate the various CQAs subfamilies present in green coffee beans extracts in the reversed-phase mode. The results showed that using a multistep and multisalt method with the aqueous layer of $\mathrm{LiCl}$ and/or $\left(\mathrm{NH}_{4}\right)_{2} \mathrm{SO}_{4}-\mathrm{KNO}_{3}$ as a mobile phase, in descending mode to mimic a conventional reversed phase gradient, and the mixture ethyl acetate-hexane as a stationary phase, the extract of green coffee beans can be effectively fractionated into subfamilies of CQAs, FQAs and diCQAs. According to the authors the manipulation of electrostriction in an aqueous solution, by changing the concentration of one or more salts, can constitute an effective method of performing a reversed-phase-like gradient elution in counter-current separations. Owing to the proposed salting-out gradients is possible the fast single-column elution of single compounds largely differing in their distribution constants without experiencing significant column bleeding.

\section{Chlorogenic acids analysis}

There are many analytical methods to identify and quantify chlorogenic acids in natural samples. According to our knowledge, however, there are no official and universally accepted methods of CQAs analysis. Therefore many laboratories develop and validate their own analysis methods, All the more that depending on the analytical technique chosen different approaches to sample preparation can be considered and, as it was stated before, nowadays there is a tendency to replace the conventional extraction techniques by newer and more effective processes. It should be also remembered that even when using the same analytical technique, different equipment and different settings can be selected. A great example of how the change of apparatus can change the result of the CQAs analysis of the same sample carried out even by the same methodology in different laboratories is the work of Craig et al. (2016). The fact that CQAs constitute a large family of compounds with various properties present in natural samples on different concentration levels, makes their analysis additionally more difficult. Hence, frequently in the course of single run, only a few CQAs representatives with very similar properties are analyzed, for example monoCQAs or diCQAs, and even an individual compound i.e. 5-CQA is subjected to analysis. Yet due to the application of modern highly selective, sensitive and accurate analytical techniques, this trend begins to change slowly towards the analysis of large numbers of CQAs in one single run. The examples of currently applied methods of CQAs analysis are summarized in Table 1. They show that liquid chromatography (LC) and capillary electrophoresis (CE) with different detection systems are the most commonly applied.

\section{Electrophoretic analysis}

Guan et al. (2006) developed a method based on capillary electrophoresis with electrochemical detection (CE-ED) to determine 5-CQA in the sweet potato. In this method the laboratory-built CE-ED system was used. A $+30 \mathrm{kV}$ high-voltage-power supply provided a separation voltage between the ends of the capillary. The inlet end of the capillary was held at a positive potential, and the outlet end was maintained at ground. All samples were injected electrokinetically, applying $18 \mathrm{kV}$ for $6 \mathrm{~s}$. A three-electrode cell system consisting of a carbon-disk working electrode, a platinum auxiliary electrode, and a SCE (saturated calomel electrode) reference electrode was used in combination with amperometric detector. By the migration time of the analyte as compared with the electropherogram of the standard solution, the active ingredient in the sweet potato samples can be identified and quantitatively determined. The results obtained by Guan et al. (2006) indicate that CE-ED is an accurate, sensitive and useful method for the quantitation of 5-CQA in the sweet potato.

Jiang et al. (2004) employed an improved capillary electrophoresis procedure for the determination of chlorogenic acid in the tobacco samples. In the procedure the analyte zone after being separated by capillary electrophoresis was determined by indirect chemiluminescence of luminol-potassium hexacyanoferrate. In this system luminal was added into the running buffer solution and introduced at the head of separation quartz capillary, and potassium 


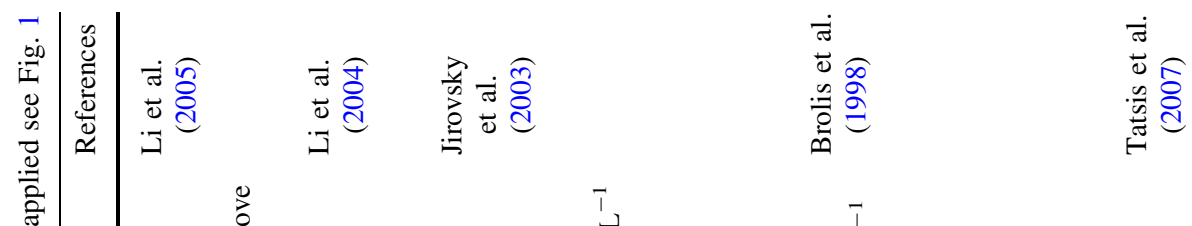

:

范

$\stackrel{Ð}{=}$

궁

한

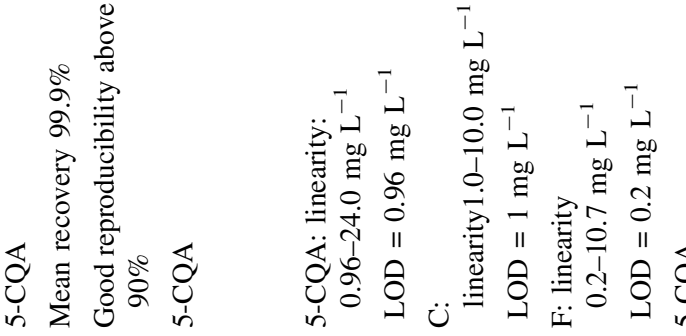

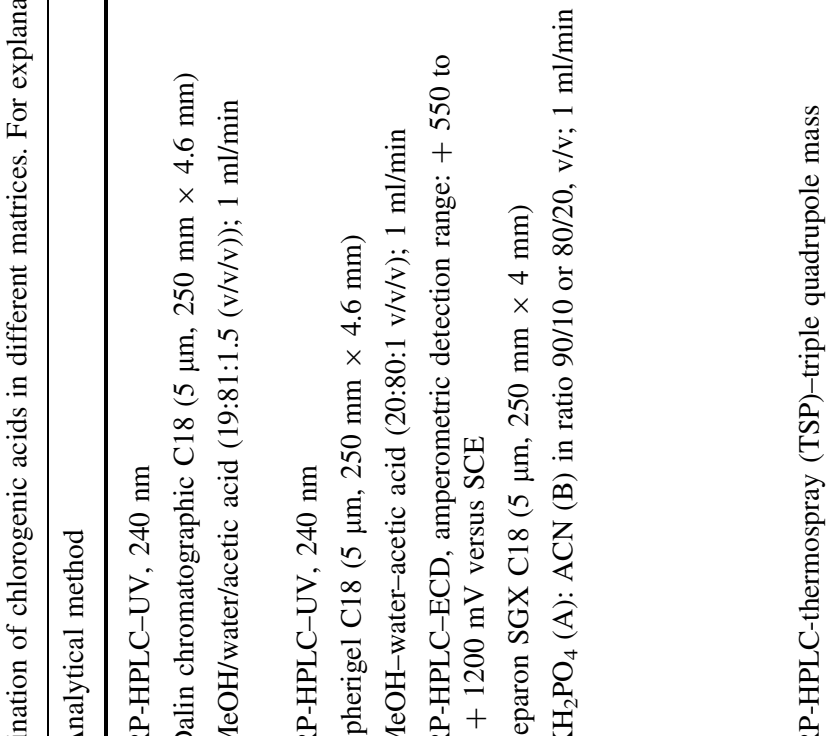

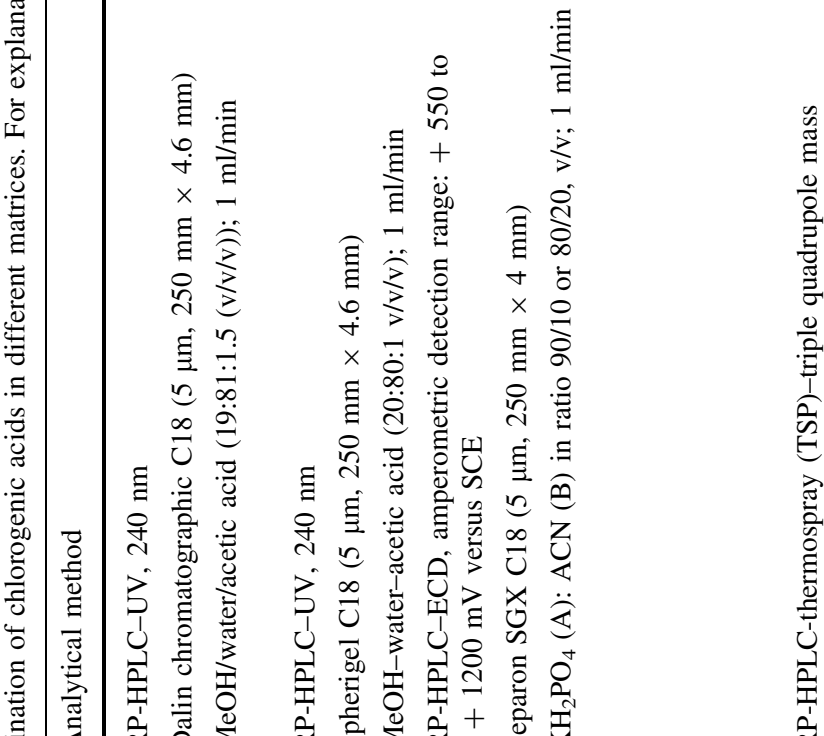

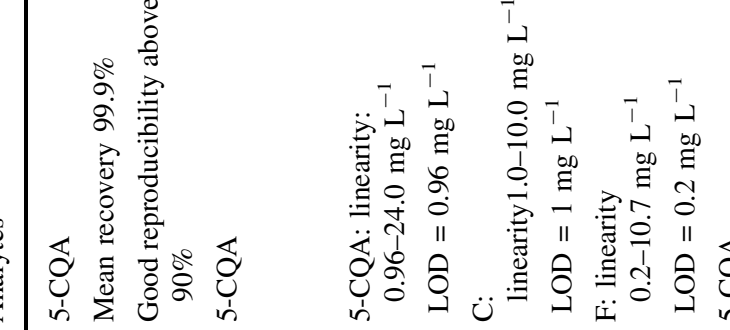

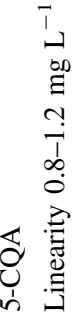

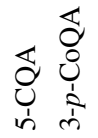

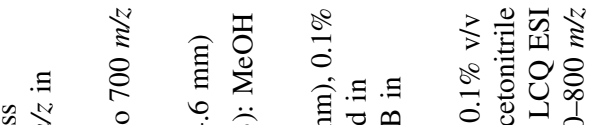

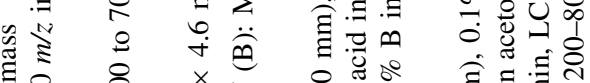

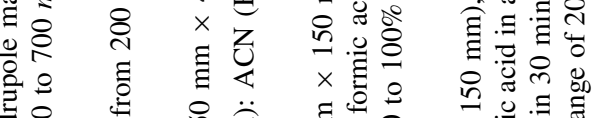

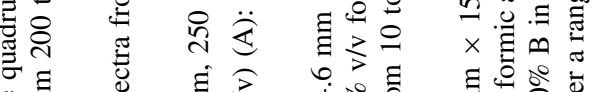

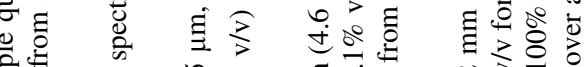

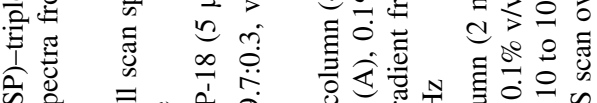

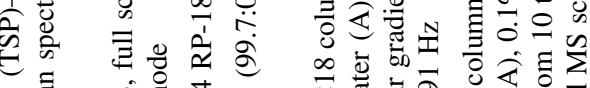

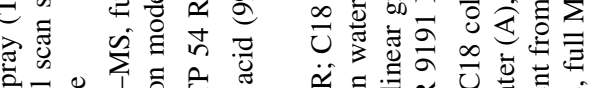

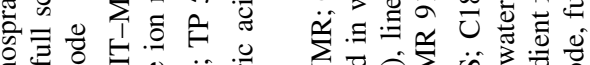

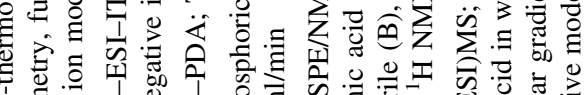

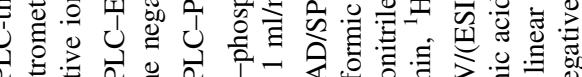

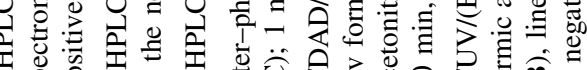

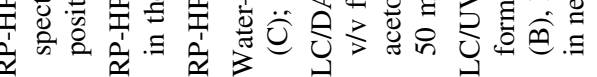

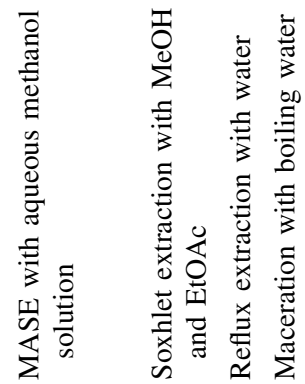

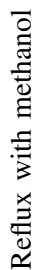

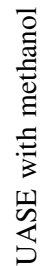

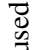

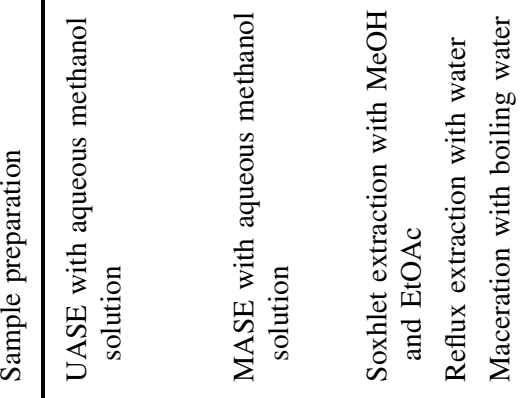

竞

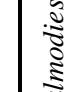

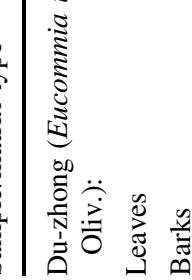

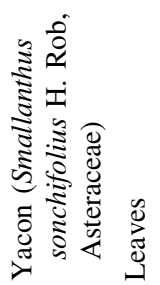

章 


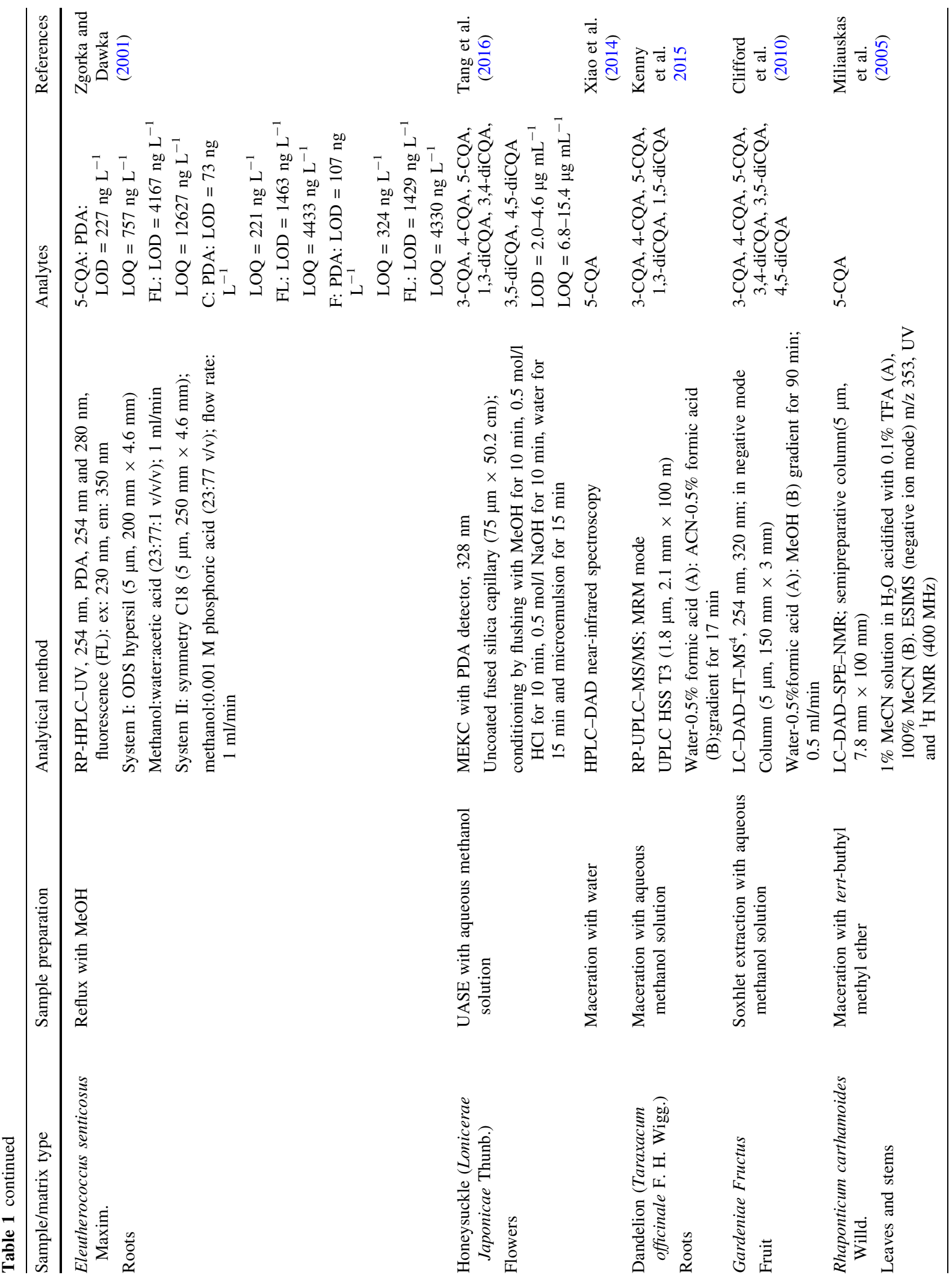




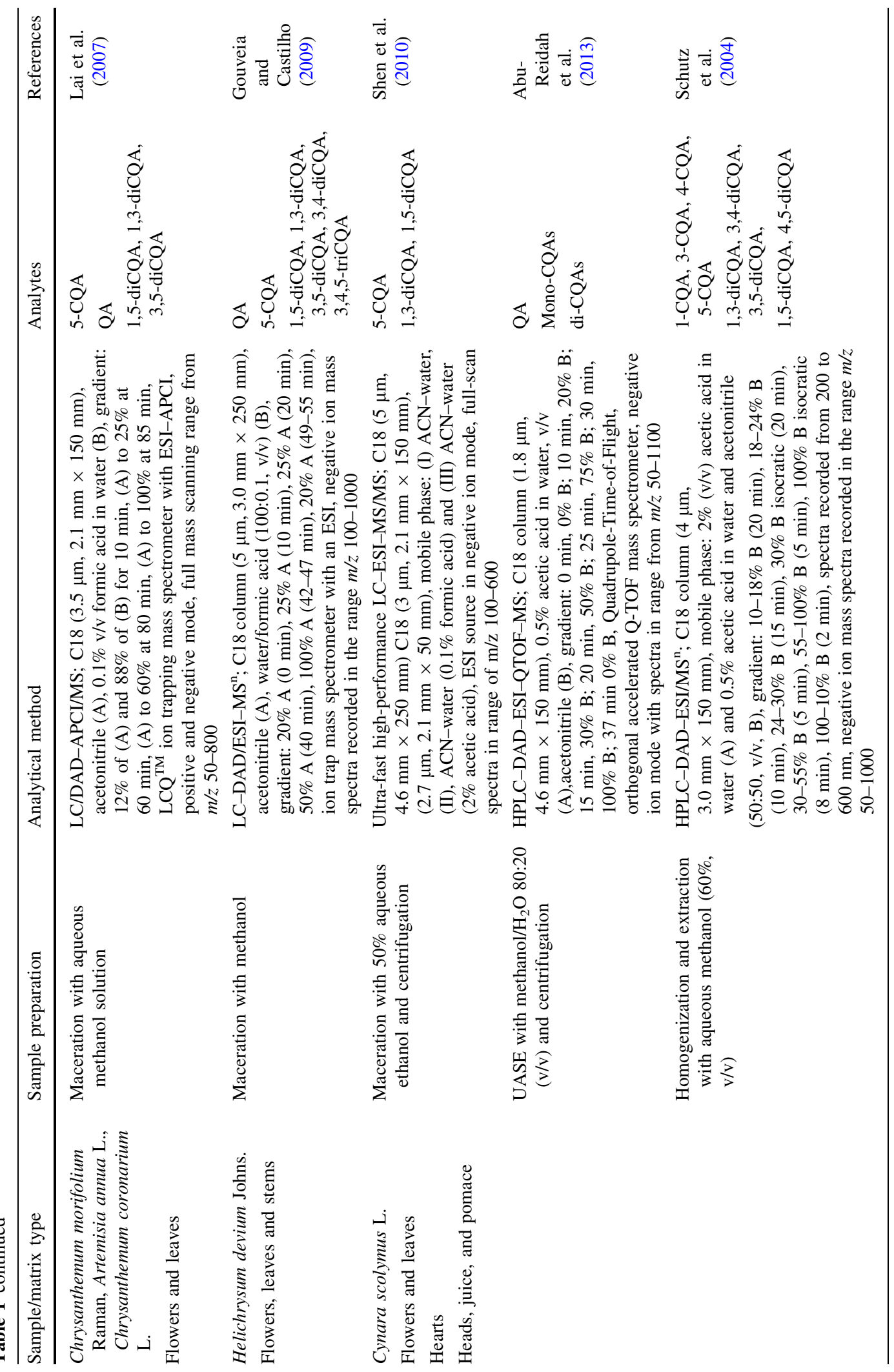




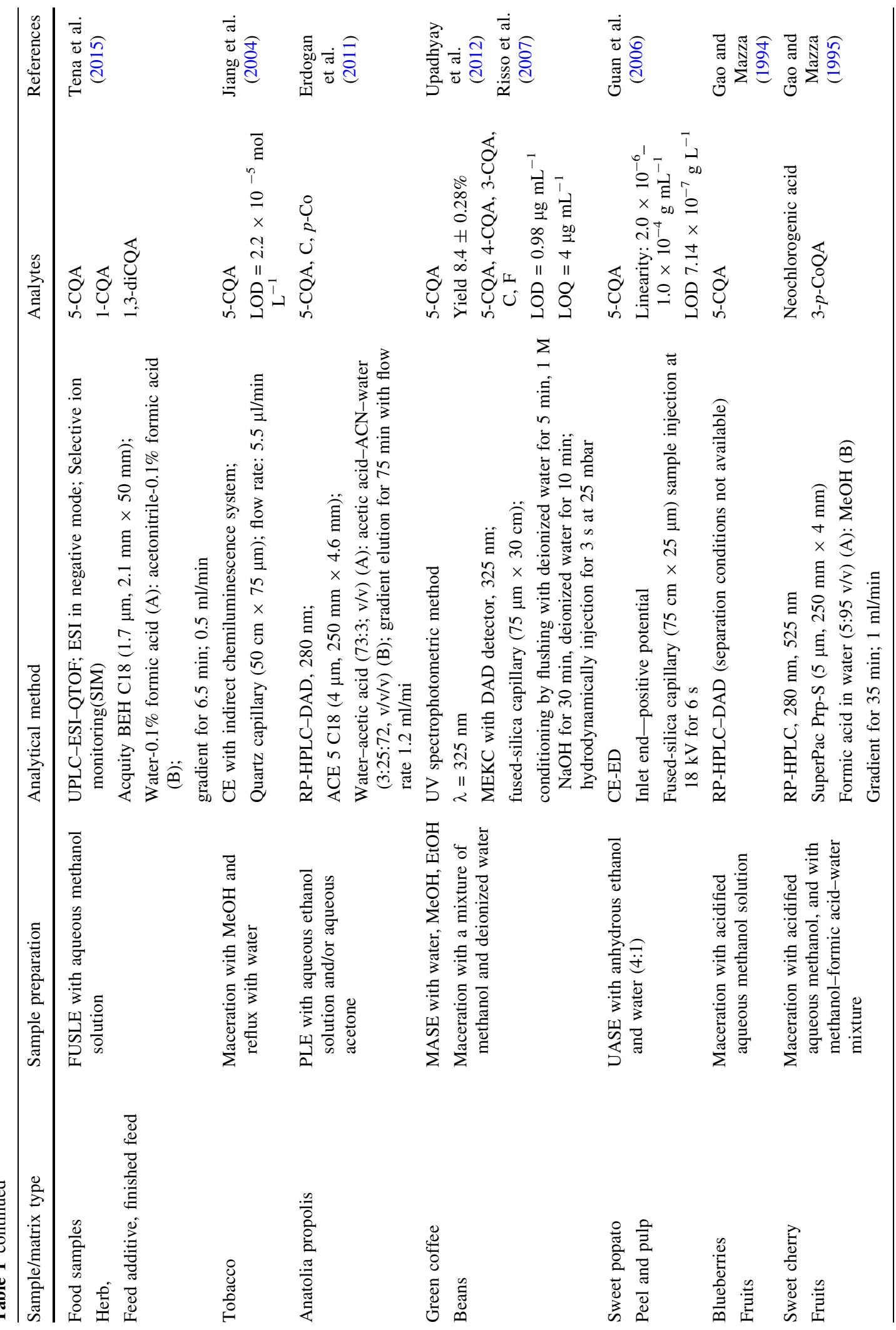




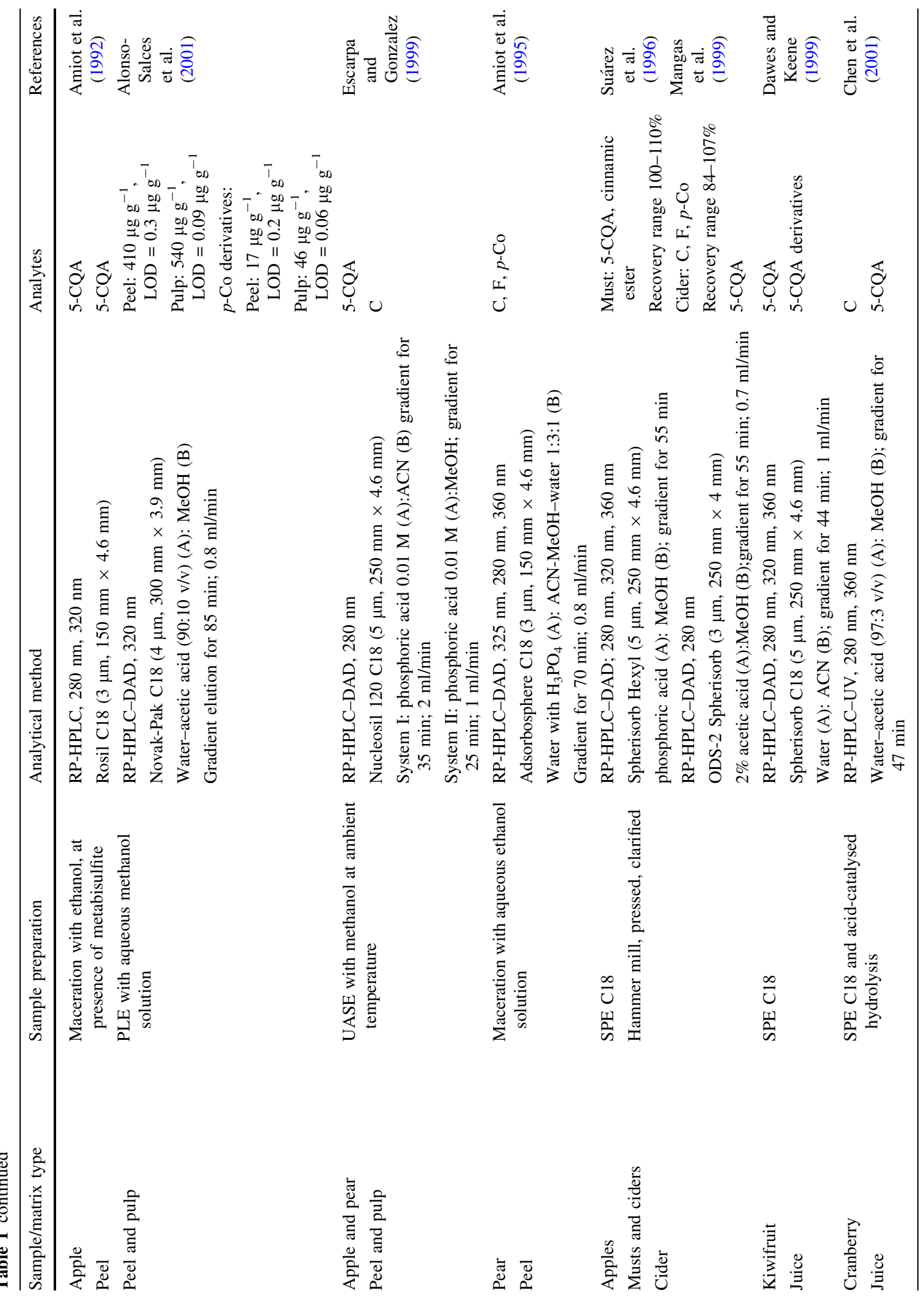




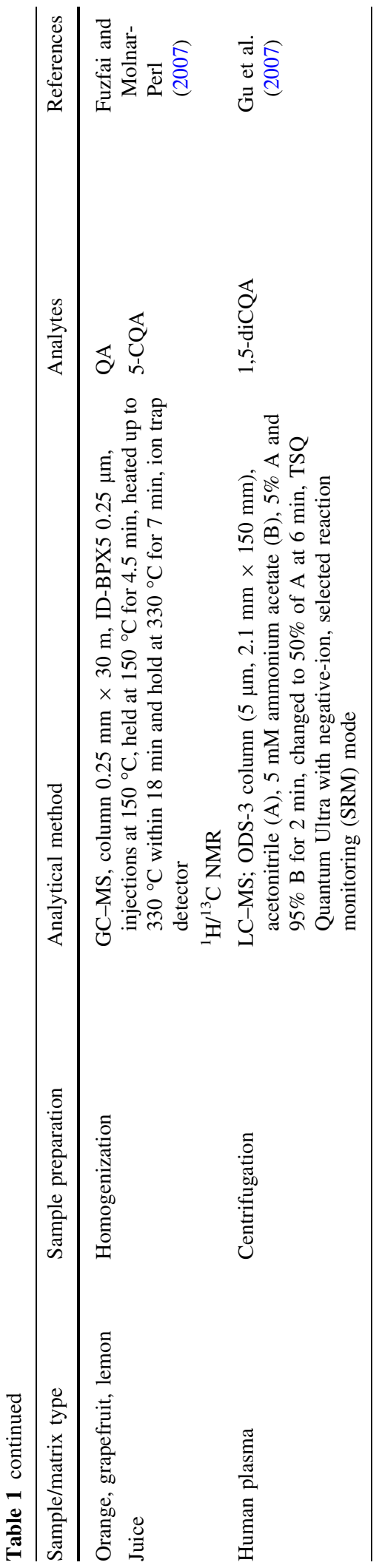

hexacyanoferrate was introduced at the end of capillary. 5-CQA in the samples was determined quantitatively by the calibration of the standard addition method and the correction of matrix background signal. According to the authors, the proposed capillary electrophoresis-chemiluminescence system can avoid the electrolysis of chemiluminescence reagent, retain the stability of chemiluminescence baseline and prolong the working time of running and electrode buffer solutions.

A special mode of capillary electrophoresis employing a microemulsion as a carrier electrolyte, so-called the microemulsion electrokinetic chromatography (MEEKC) method, in which analytes can partition between the aqueous phase of microemulsion and its oil droplets acting as a pseudostationary phase, was employed by Tang et al. (2016) for the simultaneous determination of seven CQAs acids in the honeysuckle-related products. The proposed MEEKC procedure with the PDA detection at $328 \mathrm{~nm}$, with the addition of tartrate for improving separation efficiency, was sufficient for separation and quantification of three monoCQAs and four diCQAs within $22 \mathrm{~min}$. Citing the authors of the report, the method proposed by them can be extensively used to determine individual CQA isomers in the honeysuckle or its preparations, and can provide more references for the quality evaluation and control of CQA related products.

Another modification of capillary electrophoresis i.e. micellar electrokinetic chromatography (MEKC), in which the samples are separated by differential partitioning between the micelles (pseudostationary phase) and the surrounding aqueous buffer solution acting as a mobile phase, was proposed by Risso et al. (2007) for separation of CQAs in coffee. The electrophoretic conditions consisted of the sodium dodecyl sulphate (SDS) (70 mM)-phosphate (17.6 mM)-methanol (5\%, v/v) buffer system, $\mathrm{pH}$ $2.5,22.1{ }^{\circ} \mathrm{C},-17 \mathrm{kV}$ and detection at $235 \mathrm{~nm}$. The separations were made on a fused-silica capillary with the effective and total lengths of $22.5 \mathrm{~cm}$ and $30 \mathrm{~cm}$, respectively. The method was tested with both green and roasted coffee beans, and in both cases it was proved to be effective and selective. According to the authors of the paper, the method can be used to monitor the presence of mono-CQA isomers in both natural and processed vegetables. 
Chromatographic analysis

Numerous papers have been published on qualitative and quantitative analysis of CQAs in plants by liquid chromatography (LC) coupled with the spectroscopic detection techniques, such as ultraviolet (UV), fluorescence (FL) nuclear magnetic resonance (NMR) and mass spectroscopy (MS), and even near-infrared (NIR) spectroscopy. Nowadays, the commonly observed trend of improving analytical abilities consists in using couplings with more than one separation and detection technique, e.g. LC-UV-MS, LC-MSMS, LC-NMR-MS, and the like (Jaiswal et al. 2012; Clifford et al. 2005; Deshpande et al. 2014; Gu et al. 2007; Dai et al. 2015; Bajko et al. 2016; Tena et al. 2015). In the case of plants in which CQAs are present on low concentration levels, and their precise analysis is essential, more integrated and sophisticated systems are applied that couple on-line the process of CQAs extraction with their analysis e.g. focused ultrasound solid-liquid extraction (FUSLE) coupled to LC-MS (FUSLE-LC-MS) (Tena et al.2015).

High-performance liquid chromatography (HPLC) is the most widely used analytical separation technique of CQAs. Though the more advanced form of LC i.e. ultra-performance liquid chromatography (UPLC) utilizing a sub-2-micron analytical column becomes increasingly popular for the determination of plant extract compounds, due to the improved resolution there are obtained shorter retention times and higher sensitivity (Shen et al. 2010; Tena et al. 2015). In general, LC separations of CQAs are performed by the reversed phase system (see Table 1). The most commonly applied columns are based on C18 stationary phases 0 . A typical mobile phase consists of mixtures of methanol and water. Detectors used to monitor CQAs separations are diode array detectors (DAD) or photodiode array detectors (PDA) recording at $280,320 \mathrm{and} /$ or $330 \mathrm{~nm}$ (the range of $320-330 \mathrm{~nm}$ is the range of the absorption maximum of cinnamoyl moiety) and scanning from 200 to $600 \mathrm{~nm}$. The data useful for identification of CQAs by means of LC are collected in Table 2.

It should be remembered, however, that only a few CQAs standards are available commercially, and the precise analysis of the individual CQAs by means of conventional LC-DAD (PDA) systems is not easy due to the difficulty of distinguishing between the positional isomers especially when they are present at low concentrations. Hence, nowadays most laboratories prefer the use of MS systems, and especially tandem MS, due to their higher selectivity and sensitivity. In order to improve the ionization capacity, formic acid is frequently added to the mobile phase comprising methanol as the organic modifier (see Table 1). Ionization is usually performed by electrospray ionization (ESI) source working in the negative and positive mode. Only one paper (Lai et al. 2007) selected atmospheric pressure chemical ionisation, in positive and negative mode, for analysis CQAs in three Compositae plants. Regardless of the type of ionization source, for negative mode ionization, the optimal mobile phases descibed in the literature contain a lower concentrations of formic acid (typically $0.1 \%$ ), whereas for the positive mode higher formic acid concentrations $(>0.1 \%)$. Comparing the sensitivity of both ionization modes, due to reduced chemical noise in the negative ionization mode, this mode is more sensitive than positive ionization. This is why the ESI ionization source, the most sensitive of all atmospheric pressure ionisation sources, typically operates in the negative mode. The correctness of this statement is confirmed by the data comparing the CQAs ionization efficiency of ESI and APCI in both positive and negative mode (Lai et al. 2007; Shen et al. 2010; Willems et al. 2016). For most MS applications for CQAs analysis (see Table 1), the tandem MS worked under relatively high collision energy conditions (to $47 \mathrm{eV}$ ) to generate second order mass spectra. However, for the single MS the typicall conditions are as follows: a collision energy of $35 \%$, an ionization voltage of $3.5 \mathrm{kV}$, a capillary temperature of $350 \mathrm{oC}$, a sheath gas flow rate of 65 arbitrary units, and an auxiliary gas flow rate of 10 arbitrary units. As for the most common MS analysers in the LC-based methods for the CQAs analysis in plants these are ion trap (IP), triple quadrupole (TQD) and time of flight (TOF) (Brolis et al. 1998; Gu et al. 2007; Paya et al. 2007; Shen et al. 2010; Xie et al. 2011; Jaiswal et al. 2012; Abu-Reidah et al. 2013; Dawidowicz and Typek 2010, 2017; Deshpande et al. 2014; Xue et al. 2016; Tena et al. 2015; Wianowska et al. 2015a, b). Tandem MS is very convenient for performing mass spectrometric quantification and identity confirmation, as it enables analysis in the mode of Selected Reaction Monitoring (SRM), also called Multiple Reaction Monitoring (MRM). Typically 2 or 3 MRM transitions are selected for the analite. One transition (so-called 


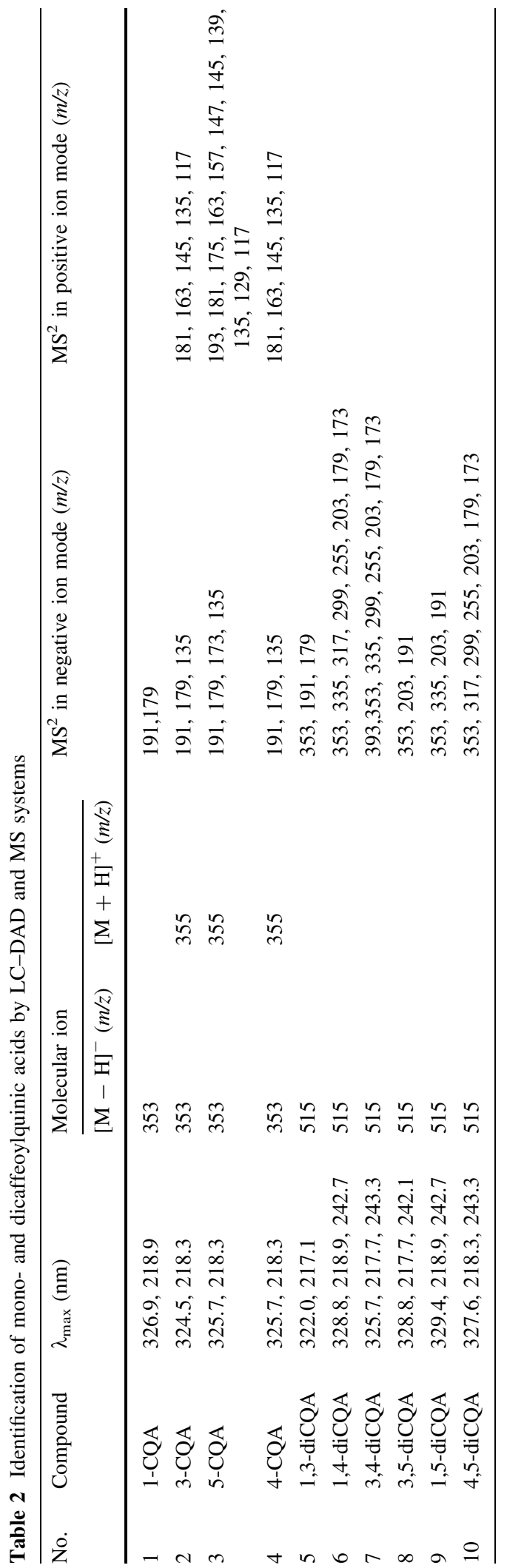

the precursor ion) is selected for quantification, the additional ones (so-called the products ions) for confirmation purposes. As both precursor ion and products ions are specified and monitored, higly selective quantitative analysis that is low in unwanted compounds can be achieved compared to single ion monitoring. Therefore the tandem MS analysis in MRM mode is ideal for sensitive and specific quantitation of a compound in very complex plant matrices.

There are many papers dealing with the application of liquid chromatography-multi-stage spectrometric identification systems $\left(\mathrm{LC}-\mathrm{MS}^{\mathrm{n}}\right)$ for qualitative and quantitative CQAs analysis in plant materials. An example can be paper (Paya et al. 2007), in which the use of UPLC-MS ${ }^{2}$ for the quantification of a series of related caffeoylquinic acid derivatives is described. In the report it was proved that due to obtaining very simple MRM chromatograms, this hyphenated technique allows for more definite identification and indeed quantification of individual isomeric compounds based on their molecular mass and MS/MS fragmentation.

Regarding the use of MS fragmentation for CQAs identification, the world of science owes much to the investigations completed in the Clifford's research group. The team investigating the fragmentation behavior of 18 CQAs, that are not substituted at position 1 , proved that the fragmentation patterns of chlorogenic acids are dependent upon the particular stereochemical relationships between the individual substituents on the quinic acid moiety (Clifford et al. 2003). In reference to the obtained results the authors stated that it is possible to discriminate between each of three isomers of p-coumaroylquinic acid, caffeoylquinic acid, feruloylquinic acid and dicaffeoylquinic acid. More importantly, on the basis of the obtained information on fragmentation patterns, the authors have prepared a hierarchical key, which has become a tool widely used by scientists to facilitate the identification process of chlorogenic acids derivatives and conjugates when standards are not available. The $\mathrm{LC}-\mathrm{MS}^{\mathrm{n}}$ identification scheme of mono- and diacylochlorogenic acids is illustrated in Fig. 2. The key stages of this protocol are as follows (Clifford et al. 2005):

$1 \mathrm{MS}^{\mathrm{n}}$ for monoacyl CQAs corresponds to $\mathrm{MS}^{\mathrm{n}+1}$ for related diacyl CQAs (As the subsequent degradation of these ions is identical, regardless 


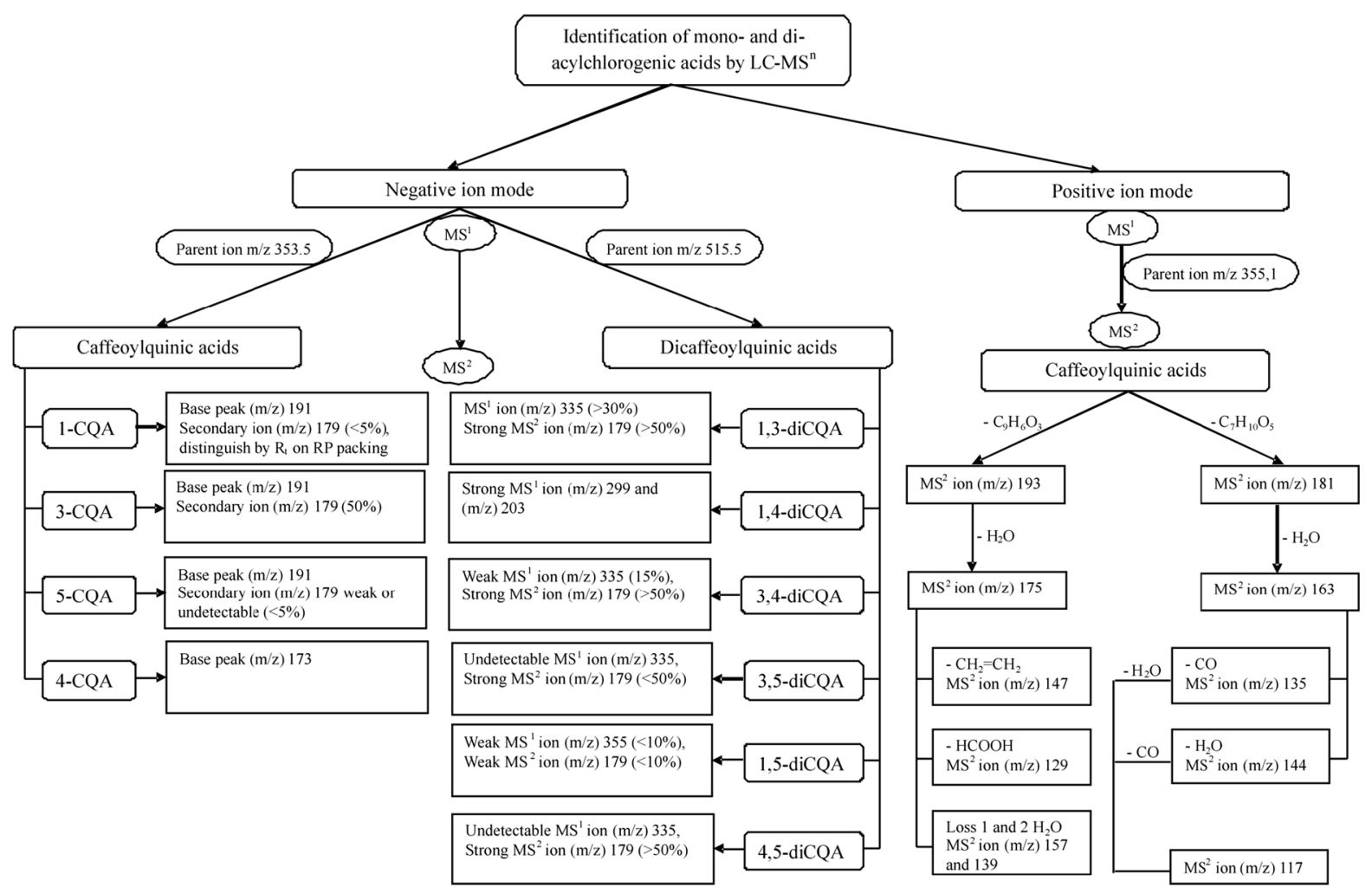

Fig. 2 LC-MS ${ }^{\mathrm{n}}$ identification scheme of mono- and diacylochlorogenic acids

of whether they have been derived from CQAs or diCQAs, to define the precise structure of the ions responsible for the diCQAs $\mathrm{MS}^{2}$ base peaks the diCQAs $\mathrm{MS}^{3}$ data should be compared with the CQAs $\mathrm{MS}^{2}$ data).

2 Individual CQAs can be distinguished based on their molecular ions $\left[\mathrm{CQA}-\mathrm{H}^{+}\right]^{-}$:

- 3-Acyl CQAs and diCQAs can be distinguished by combination of $\mathrm{MS}^{2}$ or $\mathrm{MS}^{3}$ base peak at $m / z 191$ accompanied by an intense ( $>50 \%$ base peak) $\left[\right.$ cinnamate- $\mathrm{H}^{+}$] fragment;

- 4-Acyl CQAs can be distinguished by an $\mathrm{MS}^{2}$ or $\mathrm{MS}^{3}$ base peak at $\mathrm{m} / \mathrm{z}, 173$;

- 5-Acyl CQAs can be distinguished by an $\mathrm{MS}^{2}$ or $\mathrm{MS}^{3}$ base peak at $\mathrm{m} / \mathrm{z} 191$ accompanied by a weak $\left(<5 \%\right.$ of base peak) $\left[\text { cinnamate- } \mathrm{H}^{+}\right]^{-}$ fragment.

In (Clifford et al. 2005) the developed hierarchical key has been extended to accommodate 1-caffeoylquinic acid and 1-acyl dicaffeoylquinic acids. Using this protocol and $\mathrm{LC}-\mathrm{MS}^{4}$ the authors revealed that the caffeoyl residue at position 1 is the easiest to take apart from the molecule during fragmentation, followed by residues at positions 5, 3 and 4 . Nevertheless the MS fragmentation is insufficient to distinguish 1-CQA from 5-CQA. However, the greater hydrophobicity of 5-CQA and its commercial availability ensure that these two isomers can be distinguished by the retention time on the reversed phase columns. Later the hierarchical key has been extended further for identification of 3,4,5-triacyl chlorogenic acids by Jaiswal and Kuhnert (2010). Due to the introduction of simple guidelines for regioisomer assignment, based on the successive sequencing of acyl fragmentation the researchers were able to determine eight quantitatively minor triacyl chlorogenic acids, most of which were not reported in nature previously. Finally it was reported in Clifford et al. (2008) that geometrical isomerism does not influence the fragmentation patterns of the positional isomers significantly.

There are many reports (Clifford et al. 2003, 2005, 2006, 2007a, b, 2010; Jaiswal and 
Kuhnert 2010, 2011a, b; Jaiswal et al. 2014; Matei et al. 2012) about determination of chlorogenic acids in different plants using the fragmentation patterns in the LC-MS ${ }^{\text {n }}$ systems. In Clifford et al. (2010) it was described the application of the $\mathrm{LC} / \mathrm{MS}^{4}$ system for the qualitative investigation of Gardeniae Fructus chlorogenic acids. In the applied system a LC chromatograph with DAD was interfaced with an ion-trap mass spectrometer fitted with an ESI source operating in full scan. $\mathrm{MS}^{2}, \mathrm{MS}^{3}$ and $\mathrm{MS}^{4}$ fragmenttargeted experiments were performed to focus only on the compounds producing a precursor ion at $m / 2335$, $397,497,511,543,559,573,659$, and 673 . Using this method it was possible to distinguish twenty-nine chlorogenic acids. Twenty-four of these twenty-nine CQAs were detected for the first time in this plant. Fourteen of them were not previously reported from any source.

In Clifford et al. (2006) more than thirty chlorogenic acids were identified in the aqueous-alcoholic extract of Aster ageratoides Turcz. flower buds. Among the identified compounds there were three isomers each of $p$-coumaroylquinic acid, caffeoylquinic acid, feruloylquinic acid, dicaffeoylquinic acid and diferuloylquinic acid, and six isomers each of $p$-coumaroyl-caffeoylquinic acid, $p$-coumaroyl-feruloylquinic acid and caffeoylferuloylquinic acid. Three of the six p-coumaroyl-feruloylquinic acids were not observed in nature previously. The same team of researchers profiled qualitatively herbal chrysanthemum by LC-MS $^{5}$ (Clifford et al. 2007a, b) and disclosed that apart from 5-CQA, the main chlorogenic compound, there were detected three p-coumaroylquinic acids, three feruloylquinic acids, four caffeoylquinic acids, six dicaffeoylquinic acids, and two tricaffeoylquinic acids, 13 for the first time from this source. Besides partial characterization of minor components suggested the presence of five caffeoylhexose esters and caffeic acid-4- $\beta$-D-glucose, and eight caffeoylquinic acid glycosides and 16 dicaffeoylquinic acid glycosides that were not reported in nature previously.

Studies on the CQAs composition in burdock roots (Arctium lappa L.) described in Jaiswal et al. (2011), revealed small amounts of fumaric, succinic, and malic acid-containing chlorogenic acids, of which 11 were not reported in nature previously. In reference to the results, the authors stated that despite revealing the significant differences in the MS fragmentation patterns of all CQAs regioisomers, the structurediagnostic hierarchical keys that have been developed based on fragmentation preferences induced by characteristic hydrogen bonding arrays within the gas phase ions of CQAs in tandem MS spectra, UV spectrum, retention times, and relative hydrophobicity, are very useful tools for the identification of chlorogenic acids in the complex plant matrices.

\section{Conclusions}

The objective of this review paper is to give an overview of occurrence, properties, and methods that have been developed to improve the extraction and analysis of chlorogenic acids in plants and related products, with special attention paid to 5-O-caffeoylquinic acid as the compound of the chlorogenic acids family with many beneficial health effects. Due to the potential biological activity of these compounds, recently there has been an increased interest in natural products rich in chlorogenic acids. Consequently, more efficient extraction methods, leading to getting extracts with the enhanced CQAs content and the reduced content of their degradation products, are being sought. On the other hand, taking into account the application of CQAs as pharmaceutical and food additives, the great structural similarity of CQAs and the deficiency of reference standards, more efficient and reliable analytical methods are constantly sought, in order to identify them exactly, especially when they are present at low concentrations in complex mixtures.

In the last few years a number of improvements in the strategy of extraction and analysis of CQAs in different natural samples was achieved. The application of newer, faster, more efficient and sophisticated extraction techniques, such as UASE, MASE, SFE or PLE, improved significantly the extraction yield of CQAs with significant reduction of organic solvents consumption. The overall quality of the extracts was also improved. In addition, this resulted in reduction of manipulation errors, and in consequence, it led to improved reproducibility and repeatability of the analytical methods.

There are no official and universally accepted methods of CQAs analysis in plants and related products. The large number of compounds belonging to the CQAs family and the fact that these methods need to be applicable to many various plants make this 
desire difficult to accomplish. Nevertheless nowadays two analytical techniques commonly applied for the analytical separation of CQAs can be distinguished. These are capillary electrophoresis and liquid chromatography. The latter, especially when coupled to tandem MS/MS, can be even considered as the technique that occupies the leading position for the analysis of compounds belonging to the CQAs family. This statement is supported by the fact that recently developed LC-MS ${ }^{\mathrm{n}}$ protocols, based on the determination of the same fragment ions belonging to similar structures of compounds forming the family (so-called the fragmentation patterns), greatly facilitated CQAs identification without the need to isolate pure compounds.

Open Access This article is distributed under the terms of the Creative Commons Attribution 4.0 International License (http:// creativecommons.org/licenses/by/4.0/), which permits unrestricted use, distribution, and reproduction in any medium, provided you give appropriate credit to the original author(s) and the source, provide a link to the Creative Commons license, and indicate if changes were made.

\section{References}

Abu-Reidah IM, Arraez-Roman D, Segura-Carretero A, Fernandez-Gutierrez A (2013) Extensive characterization of bioactive phenolic constituents from globe artichoke $(C y$ nara scolymus L.) by HPLC-DAD-ESI-QTOF-MS. Food Chem 141:2269-2277

Alonso-Salces RM, Korta E, Barranco A et al (2001) Pressurized liquid extraction for the determination of polyphenols in apple. J Chromatogr A 933:37-43

Amiot MJ, Tacchini M, Aubert S, Nicolas J (1992) Phenolic composition and browning susceptibility of various apple cultivars at maturity. J Food Sci 57:958-962

Amiot MJ, Tacchini M, Aubert SY, Oleszek W (1995) Influence of cultivar, maturity stage, and storage conditions on phenolic composition and enzymic browning of pear fruits. J Agric Food Chem 43:1132-1137

Azevedo ABA, Mazzafera P, Mohamed RS et al (2008) Extraction of caffeine, chlorogenic acids and lipids from green coffee beans using supercritical carbon dioxide and co-solvents. Braz J Chem Eng 25:543-552

Bajko E, Kalinowska M, Borowski P et al (2016) 5-O-Caffeoylquinic acid: a spectroscopic study and biological screening for antimicrobial activity. LWT Food Sci Technol 65:471-479

Brolis M, Gabetta B, Fuzzati N et al (1998) Identification by high-performance liquid chromatography-diode array detection-mass spectrometry and quantification by highperformance liquid chromatography-UV absorbance detection of active constituents of Hypericum perforatum. J Chromatogr A 825:9-16
Burda S, Oleszek W, Lee CY (1998) Phenolic compounds and their changes in apples during maturation and cold storage. J Agric Food Chem 38:945-948

Carabias-Martinez R, Rodriguez-Gonzalo E, Revilla-Ruiz P, Hernandez-Mendez J (2005) Pressurized liquid extraction in the analysis of food and biological samples. J Chromatogr A 1089:1-17

Chen H, Zuo Y, Deng Y (2001) Separation and determination of flavonoids and other phenolic compounds in cranberry juice by high-performance liquid chromatography. J Chromatogr A 913:387-395

Chen Y-L, Huang S-T, Sun F-M, Chiang Y-L, Chiang C-J, Tsai C-M, Weng C-J (2011) Transformation of cinnamic acid from trans- to cis-form raises a notable bactericidal and synergistic activity against multiple-drug resistant Mycobacterium tuberculosis. Eur J Pharm Sci 43:188-194

Clifford MN (1999) Review: chlorogenic acids and other cinnamates-nature, occurrence and dietary burden. J Sci Food Agric 79:362-372

Clifford MN (2000) Review: chlorogenic acids and other cinnamates-nature, occurrence, dietary burden, absorption and metabolism. J Sci Food Agric 80:1033-1043

Clifford MN, Ramirez-Martinez RJ (1990) Chlorogenic acids and purine alkaloid content of Mate (Ilex paraguariensis) leaf and beverage. Food Chem 35:13-21

Clifford MN, Walker R (1987) Letter to the editor: chlorogenic acids - confounders of coffee-serum cholesterol relationship. Food Chem 24:77-80

Clifford MN, Johnston KL, Knight S, Kuhnert N (2003) Hierarchical scheme for $\mathrm{LC}-\mathrm{MS}^{\mathrm{n}}$ identification of chlorogenic acids. J Agric Food Chem 51:2900-2911

Clifford MN, Knight S, Kuhnert N (2005) Discriminating between the six isomers of dicaffeoylquinic acid by LC$M^{n}{ }^{n}$ J Agric Food Chem 53:3821-3832

Clifford MN, Zheng W, Kuhnert N (2006) Profiling the chlorogenic acids of aster by HPLC-MS ${ }^{\mathrm{n}}$. Phytochem Anal 17:384-393

Clifford MN, Stoupi S, Kuhnert N (2007a) Profiling and characterization by $\mathrm{LC}-\mathrm{MS}^{\mathrm{n}}$ of the galloylquinic acids of green tea, tara tannin, and tannic acid. J Agric Food Chem 55:2797-2807

Clifford MN, Wu W, Kirkpatrick J, Kuhnert N (2007b) Profiling the chlorogenic acids and other caffeic acid derivatives of herbal chrysanthemum by LC-MS ${ }^{n}$. J Agric Food Chem 55:929-936

Clifford MN, Kirkpatrick J, Kuhnert N et al (2008) LC-MS ${ }^{\text {n }}$ analysis of the cis isomers of chlorogenic acids. Food Chem 106:379-385

Clifford NM, Wu W, Kirkpatrick J et al (2010) Profiling and characterization by liquid chromatography/multi-stage mass spectrometry of the chlorogenic acids in Gardeniae Fructus. Rapid Commun Mass Spectrom 24:3109-3120

Clifford MN, Jaganath IB, Ludwig IA, Crozier A (2017) Chlorogenic acids and the acyl-quinic acids: discovery, biosynthesis, bioabailability and bioactivity. Nat Prod Rep 34:1391-1421

Craig AP, Fields C, Liang N et al (2016) Performance review of a fast HPLC-UV method for the quantification of chlorogenic acids in green coffee bean extract. Talanta 154:481-485 
Dai G, Ma S, Sun B et al (2015) Simultaneous determination of 1,3-dicaffeoylquinic acid and caffeic acid in rat plasma by liquid chromatography/tandem mass spectrometry and its application to a pharmacokinetic study. Anal Methods 7:3587-3592

Dawes HM, Keene JB (1999) Phenolic composition of kiwifruit juice. J Agric Food Chem 47:2398-2403

Dawidowicz AL, Typek R (2010) Thermal stability of 5-Ocaffeoylquinic acid in aqueous solutions at different heating conditions. J Agric Food Chem 58:12578-12584

Dawidowicz AL, Typek R (2015) Thermal transformation of trans-5-O-caffeoylquinic acid (trans-5-CQA) in alcoholic solutions. Food Chem 167:52-62

Dawidowicz AL, Typek R (2017) Transformation of chlorogenic acids during the coffee beans roasting process. Eur Food Res Technol 243:379-390

Dawidowicz AL, Wianowska D (2005a) PLE in the analysis of plant compounds. II. One-cycle PLE in determining total amount of analyte in plant material. J Pharm Biomed Anal 37:1161-1165

Dawidowicz AL, Wianowska D (2005b) PLE in the analysis of plant compounds. Part I. The application of PLE for HPLC analysis of caffeine in green tea leaves. J Pharm Biomed Anal 37:1155-1159

Dawidowicz AL, Wianowska D (2009) Application of the matrix solid-phase dispersion technique for the HPLC analysis of rutin in Sambucus nigra L.: the linear correlation of the matrix solid-phase dispersion process. J Chromatogr Sci 47:914-918

Dawidowicz AL, Rado E, Wianowska D (2009) Static and dynamic superheated water extraction of essential oil components from Thymus vulgaris L. J Sep Sci 32:3034-3042

Dawidowicz AL, Wianowska D, Rado E (2011) Matrix solidphase dispersion with sand in chromatographic analysis of essential oils in herbs. Phytochem Anal 22:51-58

Dawidowicz AL, Czapczyńska NB, Wianowska D (2013) Relevance of the sea sand disruption method (SSDM) for the biometrical differentiation of the essential oil composition from conifers. Chem Biodivers 10:241-250

Dent M, Dragovic-Uzelac V, Elez-Garofulic I et al (2015) Comparison of conventional and ultrasound-assisted extraction techniques on mass fraction of phenolic compounds from sage (Salvia officinalis L.). Chem Biochem Eng Q 29:475-484

Deshpande S, Jaiswal R, Matei MF, Kuhnert N (2014) Investigation of acyl migration in mono- and dicaffeoylquinic acids under aqueous basic, aqueous acidic, and dry roasting conditions. J Agric Food Chem 62:9160-9170

El-Seedi HR, El-Said AMA, Khalifa SAM, Göransson U, Bohlin L, Borg-Karlson A-K, Verpoorte R (2012) Biosynthesis, natural sources, dietary intake, pharmacokinetic properties, and biological activities of hydroxycinnamic acids. J Agric Food Chem 60:10877-10895

Erdogan S, Ates B, Durmaz G et al (2011) Pressurized liquid extraction of phenolic compounds from Anatolia propolis and their radical scavenging capacities. Food Chem Toxicol 49:1592-1597

Escarpa A, Gonzalez MC (1999) Fast separation of (poly)phenolic compounds from apples and pears by high- performance liquid chromatography with diode-array detection. J Chromatogr A 830:301-309

Eskilsson CS, Bjorklund E (2000) Analytical-scale microwaveassisted extraction. J Chromatogr A 902:227-250

Farah A, De Paulis T, Trugo LC, Martin PR (2005) Effect of roasting on the formation of chlorogenic acid lactones in coffee. J Agric Food Chem 53:1505-1513

Fuzfai Z, Molnar-Perl I (2007) Gas chromatographic-mass spectrometric fragmentation study of flavonoids as their trimethylsilyl derivatives: analysis of flavonoids, sugars, carboxylic and amino acids in model systems and in citrus fruits. J Chromatogr A 1149:88-101

Galati G, O’brien PJ (2004) Potential toxicity of flavonoids and other dietary phenolics: significance for their chemopreventive and anticancer properties. Free Radic Biol Med 37:287-303

Gao L, Mazza G (1994) Quantitation and distribution of simple and acylated anthocyanins and other phenolics in blueberries. J Food Sci 59:1057-1059

Gao L, Mazza G (1995) Characterization, quantitation, and distribution of anthocyanins and colorless phenolics in sweet cherries. J Agric Food Chem 43:343-346

Gouveia CS, Castilho CP (2009) Analysis of phenolic compounds from different morphological parts of Helichrysum devium by liquid chromatography with on-line UV and electrospray ionization mass spectrometric detection. Rapid Commun Mass Spectrom 23:3939-3953

Gu R, Dou G, Wang J et al (2007) Simultaneous determination of 1,5-dicaffeoylquinic acid and its active metabolites in human plasma by liquid chromatography-tandem mass spectrometry for pharmacokinetic studies. J Chromatogr B 852:85-91

Guan Y, Wu T, Lin M, Ye J (2006) Determination of pharmacologically active ingredients in Sweet Potato (Ipomoea batatas L.) by capillary electrophoresis with electrochemical detection. J Agric Food Chem 54:24-28

Hanson KR (1965) Chlorogenic acid biosynthesis. Chemical synthesis and properties of the Mono- $O$-cinnamoylquinic acids. Biochemistry 4:2719-2731

Jaiswal R, Kuhnert N (2010) Hierarchical scheme for liquid chromatography/multi-stage spectrometric identification of 3,4,5-triacyl chlorogenic acids in green Robusta coffee beans. Rapid Commun Mass Spectrom 24:2283-2294

Jaiswal R, Kuhnert N (2011a) Identification and characterization of two new derivatives of chlorogenic acids in Arnica (Arnica montana L.) flowers by high-performance liquid chromatography/tandem mass spectrometr. J Agric Food Chem 59:4033-4039

Jaiswal R, Kuhnert N (2011b) Identification and characterization of five new classes of chlorogenic acids in burdock (Arctium lappa L.) roots by liquid chromatography/tandem mass spectrometry. Food Funct 2:63-71

Jaiswal R, Kiprotich J, Kuhnert N (2011) Determination of the hydroxycinnamate of 12 members of the Asteraceae family. Phytochemistry 72:781-790

Jaiswal R, Matei MF, Golon A et al (2012) Understanding the fate of chlorogenic acids in coffee roasting using mass spectrometry based targeted and non-targeted analytical strategies. Food Funct 3:976-984

Jaiswal R, Müller H, Müller A et al (2014) Identification and characterization of chlorogenic acids, chlorogenic 
acidglycosides and flavonoids from Lonicera henryi $L$. (Caprifoliaceae) leaves by LC-MS ${ }^{\mathrm{n}}$. Phytochemistry 108:252-263

Jiang H-L, He Y-Z, Zhao H-Z, Hu Y-Y (2004) Determination of chlorogenic acid and rutin in cigarettes by an improved capillary electrophoresis indirect chemiluminescence system. Anal Chim Acta 512:111-119

Jirovsky D, Horakova D, Kotoucek M et al (2003) Analysis of phenolic acids in plant materials using HPLC with amperometric detection at a platinum tubular electrode. J Sep Sci 26:739-742

Johnston KL, Clifford MN, Morgan LM (2003) Coffee acutely modifies gastrointestinal hormone secretion and glucose tolerance in humans: glycemic effects of chlorogenic acid and caffeine. Am J Clin Nutr 78:728-733

Kaufmann B, Christen P (2002) Recent extraction techniques for natural products: microwave-assisted extraction and pressurised solvent extraction. Phytochem Anal 13:105-113

Kenny O, Smyth JT, Hewage MC, Brunton PN (2015) Quantitative UPLC-MS/MS analysis of chlorogenic acid derivatives in antioxidant fractionates from dandelion (Taraxacum officinale) root. Int J Food Sci Technol 50:766-773

Khaw K-Y, Parat M-O, Shaw PN et al (2017) Solvent supercritical fluid technologies to extract bioactive compounds from natural sources: a Review. Molecules 22:1186-1208

Kweon MH, Hwang HJ, Sung HC (2001) Identification and antioxidant activity of novel chlorogenic acid derivatives from bamboo (Phyllostachys edulis). J Agric Food Chem 49:4646-4655

Kwon HC, Jung CM, Shin CG et al (2000) A new caffeoyl quinic acid from Aster scaber and its inhibitory activity against human immunodeficiency virus-1 (HIV-1) integrase. Chem Pharm Bull 48:1796-1798

Lai J-P, Lim YH, Su J et al (2007) Identification and characterization of major flavonoids and caffeoylquinic acids in three Compositae plants by LC/DAD-APCI/MS. J Chromatogr B 848:215-225

Lattanzio V, Cicco N, Linsalata V (2005) Antioxidant activities of artichoke phenolics. Acta Hortic 681:421-428

Lattanzio V, Kroon PA, Linsalata V, Cardinali A (2009) Globe artichoke: a functional food and source of nutraceutical ingredients. J Funct Foods 1:131-144

Li H, Chen B, Zhang Z, Yao S (2004) Focused microwaveassisted solvent extraction and HPLC determination of effective constituents in Eucommia ulmodies Oliv. (E. ulmodies). Talanta 63:659-665

Li H, Chen B, Yao S (2005) Application of ultrasonic technique for extraction chlorogenic acid from Eucommia umodies Oliv. (E. ulmodies). Ultrason Sonochem 12:295-300

Li YJ, Zhang C-F, Ding G et al (2015) Investigating the thermal stability of six caffeoylquinic acids employing rapid-resolution liquid chromatography with quadrupole time-offlight tandem mass spectrometry. Eur Food Res Technol 240:1225-1234

Lotito SB, Frei B (2006) Consumption of flavonoid-rich foods and increased plasma antioxidant capacity in humans: cause, consequence, or epiphenomenon? Free Radic Biol Med 41:1727-1746
Lou X, Janssen H-G, Crames CA (1997) Parameters affecting the accelerated solvent extraction of polymeric samples. Anal Chem 69:1598-1603

Ludwig LA, Clifford MN, Lean MEJ et al (2014) Coffee: biochemistry and potential impact on health. Food Funct 5:1695-1717

Luthria DL (2008) Influence of experimental conditions on the extraction of phenolic compounds from parsley (Petroselinum crispum) flakes using a pressurized liquid extractor. Food Chem 107:745-752

Machmudah S, Kitada K, Sasaki M et al (2011) Simultaneous extraction and separation process for coffee beans with supercritical $\mathrm{CO}_{2}$ and water. Ind Eng Chem Res 50:2227-2235

Mangas JJ, Rodríguez R, Suárez B et al (1999) Study of the phenolic profile of cider apple cultivars at maturity by multivariate techniques. J Agric Food Chem 47:4046-4052

Martinez-Moral MP, Tena MT (2011) Focused ultrasound solidliquid extraction and selective pressurized liquid extraction to determine bisphenol A and alylphenols in sewage sludge by gas chromatography-mass spectrometry. J Sep Sci 34:2513-2522

Matei MF, Jaiswal R, Kuhnert N (2012) Investigating the chemical changes of chlorogenic acids during coffee brewing: conjugate addition of water to the olefinic moiety of chlorogenic acids and their quinides. J Agric Food Chem 60:12105-12115

Mazzafera P (1997) Mate drinking: caffeine and phenolic acid intake. Food Chem 60:67-71

Miliauskas G, Van Beek TAW, Venskutonis PRP, Sudholter EJR (2005) Identification of radical scavenging compounds in Rhaponticum carthamoides by means of LCDAD-SPE-NMR. J Nat Prod 68:168-172

Mustafa A, Turner C (2011) Pressurized liquid extraction as a green approach in food and herbal plants extraction: a review. Anal Chim Acta 703:8-18

Oniszczuk A, Podgórski R, Oniszczuk T, Waksmundzka-Hajnos M (2014) Extraction methods for the determination of phenolic compounds from Equisetum arvense L. herb. Ind Crops Prod 61:377-381

Panizzi L, Scarpati ML (1954) Constitution of cynarine, the active principle of the artichoke. Nature 174:1062-1063

Paya P, Anastassiades M, Mack D et al (2007) Analysis of pesticides residues using the quick easy cheap effective rugged and safe (QuEChERS) pesticide multiresidue method in combination with gas and liquid chromatography and tandem mass spectrometric detection. Anal Bioanal Chem 389:1697-1714

Risso EM, Peres GR, Amaya-Farfrian J (2007) Determination of phenolic acids in coffee by micellar electrokinetic chromatography. Food Chem 105:1578-1582

Ritter G, Hagenauer-Hener U, Dietrich H (1995) The phenolic substances of Sorbus domestica $\mathrm{L}$ and their importance for the quality of apple wines. In: Brouillard R, Jay M, Scalbert A (eds) Polyphenols 94. XVIIe Journees Internationales Groupe Polyphenols. INRA Editions, Paris, p 235

Romero-Gonzalez RR, Verpoorte R (2009) Salting-out gradients in centrifugal partition chromatography for the isolation of chlorogenic acids from green coffee beans. J Chromatogr A 1216:4245-4251 
Routray W, Orsat V (2012) Microwave-assisted extraction of flavonoids: a review. Food Bioproc Technol 5:409-424

Saleh IA, Vinatoru M, Mason TJ, Abdel-Azim NS, Aboutabl EA, Hammouda FM (2016) A possible general mechanism for ultrasound-assisted extraction (UAE) suggested from the results of UAE of chlorogenic acid from Cynara scolymus L. (artichoke) leaves. Ultrason Sonochem 31:330-336

Schutz K, Kammerer D, Carle R, Schieber A (2004) Identification and quantification of caffeoylquinic acids and flavonoids from artichoke (Cynara scolymus L.) heads, juice, and pomace by HPLC-DAD-ESI/MS ${ }^{\mathrm{n}}$. J Agric Food Chem 52:4090-4096

Shearer J, Farah A, De Paulis T et al (2003) Quinides of roasted coffee enhance insulin action in conscious rats. J Nutr 133:3529-3532

Shen Q, Dai Z, Lu Y (2010) Rapid determination of caffeoylquinic acid derivatives in Cynara scolymus L. by ultra-fast liquid chromatography/tandem mass spectrometry based on a fused core C18 column. J Sep Sci 33:3152-3158

Spanos GA, Wrolstad RE (1992) Phenolics of apple, pear, and white grape juices and their changes with processing and storage: a review. J Agric Food Chem 40:1478-1487

Srinath D, Maheswari UK (2016) Ultrasound technology in food processing: a review. Int J Curr Adv Res 5:778-783

Suárez B, Picinelli A, Mangas JJ (1996) Solid-phase extraction and high-performance liquid chromatographic determination of polyphenols in apple musts and cider. J Chromatogr A 727:203-209

Tang B, Guo D, Li Y et al (2016) Separation and quantitation of isomeric caffeoylquinic acids in honeysuckle products by low-pH microemulsion electrokinetic chromatography using tartrate as a chiral selector. Anal Methods 8:189-196

Tatsis EC, Boeren S, Exarchou V (2007) Identification of the major constituents of Hypericum perforatum by LC/SPE/ NMR and/or LC/MS. Phytochemistry 68:383-393

Teixeira DM, Da Costa CT (2005) Novel methods to extract flavanones and xanthones from the root bark of Maclura pomifera. J Chromatogr A 1062:175-181

Teixeira DM, Patão FF, Coelho AV, Da Costa CT (2006) Comparison between sample disruption methods and solid-liquid extraction (SLE) to extract phenolic compounds from Ficus carica leaves. J Chromatogr A 1103:22-28

Tena MT, Martinez-Moral MP, Cardozo PW (2015) Determination of caffeoylquinic acids in feed and related products by focused ultrasound solid-liquid extraction and ultrahigh performance liquid chromatography-mass spectrometry. J Chromatogr A 1400:1-9

Torres-Mancera MT, Baqueiro-Pena I, Figueroa-Montero A et al (2013) Biotransformation and improved enzymatic extraction of chlorogenic acid from coffee pulp by filamentous fungi. Biotechnol Prog 29:337-345

Upadhyay R, Rao LJM (2013) An outlook on chlorogenic acids-occurrence, chemistry, technology, and biological activities. Crit Rev Food Sci Nutr 53:968-984

Upadhyay R, Ramalakshmi K, Rao LJM (2012) Microwaveassisted extraction of chlorogenic acids from green coffee beans. Food Chem 130:184-188
Vinatoru M, Mason TJ, Calinescu I (2017) Ultrasonically assisted extraction (UAE) and microwave assisted extraction (MAE) of functional compounds from plant materials. TrAC Trend Anal Chem 97:159-178

Waksmundzka-Hajnos M, Petruczynik A, Dragan A et al (2004) Influence of the extraction mode on the coumarines content in Archangelica officinalis fruit extract. Phytochem Anal 15:313-319

Wianowska D (2014a) The influence of purge times on the yields of essential oils components extracted from plants by pressurized liquid extraction. J AOAC Int 97:1310-1316

Wianowska D (2014b) Hydrolytical instability of hydroxyanthraquinone glycosides in pressurized liquid extraction. Anal Bioanal Chem 406:3219-3227

Wianowska D (2015) Application of sea sand disruption method for HPLC determination of quercetin in plants. J Liq Chromatogr Relat Technol 38:1037-1043

Wianowska D, Dawidowicz AL (2016a) Can matrix solid phase dispersion (MSPD) be more simplified? Application of solventless MSPD sample preparation method for GC-MS and GC-FID analysis of plant essential oil components. Talanta 151:179-182

Wianowska D, Dawidowicz AL (2016b) Effect of water content in extraction mixture on the pressurized liquid extraction efficiency-stability of quercetin 4 -glucoside during extraction from onions. J AOAC Int 99:744-749

Wianowska D, Wiśniewski M (2015) Simplified procedure of silymarin extraction from Silybium Marianum L. Gaertner. J Chromatogr Sci 53:366-372

Wianowska D, Hajnos MŁ, Dawidowicz AL et al (2009) Extraction methods of 10-deacetylbaccatin III, paclitaxel and cephalomannine from Taxus baccata L. twigs: a comparison (Comparison of extraction methods). J Liq Chromatogr Relat Technol 32:589-601

Wianowska D, Typek R, Dawidowicz AL (2015a) How to eliminate the formation of chlorogenic acids artefacts during plants analysis? Sea sand disruption method (SSDM) in the HPLC analysis of chlorogenic acids and their native derivatives in plants. Phytochemistry 117:489-499

Wianowska D, Typek R, Dawidowicz AL (2015b) Chlorogenic acid stability in pressurized liquid extraction conditions. J AOAC Int 98:415-421

Wianowska D, Dawidowicz AL, Bernacik K, Typek R (2017) Determining the true content of quercetin and its derivatives in plants. Eur Food Res Technol 243:27-40

Willems JL, Khamis MM, Saeid WM et al (2016) Analysis of a series of chlorogenic acid isomers using differential ion mobility and tandem mass spectrometry. Anal Chim Acta 933:164-174

Wu Y, Liu B, Chang Y et al (2015) Optimization of modified supercritical $\mathrm{CO}_{2}$ extraction of chlorogenic acid from the flower buds of Lonicera japonica Thunb and determination of antioxidant activity of the extracts. J Liq Chromatogr Relat Technol 38:443-450

Xiao X, Ma J, Ge F et al (2014) Application of near-infrared spectroscopy for the rapid analysis of Lonicerae Japonicae Flos solution extracted by water. J Innov Opt Health Sci 7(1350063):1-9 
Xie C, Yu K, Zhong D et al (2011) Investigation of isomeric transformations of chlorogenic acid in buffers and biological matrixes by ultraperformance liquid chromatography coupled with hybrid quadrupole/ion mobility/ orthogonal acceleration time-of-flight mass spectrometry. J Agric Food Chem 59:11078-11087

Xu J-J, Cao J, Peng L-Q et al (2016) Characterization and determination of isomers in plants using tracematrix solid phase dispersion via ultrahigh performance liquidchromatography coupled with an ultraviolet detector and quadrupoletime-of-flight tandem mass spectrometry. J Chromatogr A 1436:6472
Xue M, Shi H, Zhang J et al (2016) Stability and degradation of caffeoylquinic acids under different storage conditions studied by high-performance liquid chromatography with photo diode array detection and high-performance liquid chromatography with electrospray ionization collision-induced dissociation tandem mass spectrometry. Molecules 21:948-961

Zgorka G, Dawka S (2001) Application of conventional UV, photo-diode array (PDA) and fluorescence (FL) detection to analysis of phenolic acid in plant material and pharmaceutical preparations. J Pharm Biomed Anal 24:1065-1072 